\title{
xGASS: H I Fueling of Star Formation in Disk-dominated Galaxies
}

\author{
Jing Wang ${ }^{1}$, Barbara Catinella ${ }^{2,3}$ (1) , Amélie Saintonge ${ }^{4}$ (1) Zhizheng Pan $^{5}$ (1) Paolo Serra $^{6}$, and Li Shao ${ }^{7}$ \\ ${ }^{1}$ Kavli Institute for Astronomy and Astrophysics, Peking University, Beijing 100871, People's Republic of China \\ ${ }^{2}$ International Centre for Radio Astronomy Research (ICRAR), University of Western Australia, Crawley, WA 6009, Australia \\ ${ }^{3}$ Australian Research Council, Centre of Excellence for All Sky Astrophysics in 3 Dimensions (ASTRO 3D), Australia \\ ${ }^{4}$ Department of Physics \& Astronomy, University College London, Gower Place, London WC1E 6BT, UK \\ ${ }^{5}$ Purple Mountain Observatory, Chinese Academy of Sciences, 2 West-Beijing Road, Nanjing 210008, People's Republic of China \\ ${ }^{6}$ INAF, Osservatorio Astronomico di Cagliari, Via della Scienza 5, I-09047 Selargius, CA, Italy \\ ${ }^{7}$ National Astronomical Observatories, Chinese Academy of Sciences, 20A Datun Road, Chaoyang District, Beijing, People's Republic of China \\ Received 2019 October 21; revised 2019 December 11; accepted 2020 January 6; published 2020 February 12
}

\begin{abstract}
We introduce a method to estimate the H I mass within the optical radius of disk galaxies from integrated $\mathrm{H} \mathrm{I}$ spectra, with an uncertainty of 0.09 dex. We use these estimates to study how inner H I fuels star formation in latetype disk galaxies. We find that star formation rate (SFR) at a given stellar mass $\left(M_{*}\right)$ is well correlated with the inner H I surface density $\left(\Sigma_{\mathrm{H} \text { I,in }}\right)$ and inner H I mass-to-stellar mass ratio. For the massive $\left(M_{*}>10^{10} M_{\odot}\right)$ disk galaxies, higher SFR at a given stellar mass is also related to higher efficiency of converting inner $\mathrm{H}$ I to molecular gas, but no such correlation is found for the total $\mathrm{H} \mathrm{I}$ mass. The highest $\Sigma_{\mathrm{H} \text { I,in }}$ and the fastest depletion of the total neutral gas within the optical disks are found in the most compact and star-forming disk galaxies at a given stellar mass. These results highlight the important role of inner $\mathrm{H} \mathrm{I}$ as an intermediate step of fueling star formation in disk galaxies.
\end{abstract}

Unified Astronomy Thesaurus concepts: Late-type galaxies (907); Interstellar atomic gas (833); Galaxy evolution (594)

\section{Introduction}

Galaxies in the low- and high-redshift universe are distributed in remarkably regular patterns in the parameter space of star formation rate (SFR) versus stellar mass $\left(M_{*}\right.$; Noeske et al. 2007; Schiminovich et al. 2007; Elbaz et al. 2011; Whitaker et al. 2012, 2014; Speagle et al. 2014; Tacconi et al. 2018). Massive $\left(M_{*}>10^{9} M_{\odot}\right)$ star-forming galaxies are concentrated in a tight relation between $\log$ SFR and $\log M_{*}$, with a slope less than unity and a typical scatter of $0.3-0.4$ dex in SFR throughout the relation (Noeske et al. 2007; Speagle et al. 2014; Whitaker et al. 2014). This tight relation is referred to as the star-forming main sequence (SFMS). The less starforming galaxies show a broad range of SFR at a fixed $M_{*}$ below the SFMS.

Theories predict that the scatter of the SFMS is caused by the oscillation of galaxies around the SFMS with time, which is driven by a balance between cold gas replenishment and depletion in their inner disk (Dekel \& Burkert 2014; Tacchella et al. 2015; Zolotov et al. 2015). If replenishment is quicker than depletion, the central gas density increases, the SFR rises above the SFMS, and the central stellar mass concentration goes up. This process continues until the very high central gas surface density triggers vigorous star formation followed by strong feedback that quickly depletes the gas. This process of central gas, SFR, and stellar density building up until the onset of quick depletion is called compaction (Dekel \& Burkert 2014; Tacchella et al. 2015; Zolotov et al. 2015). At the end of compaction, the SFR drops below the SFMS, until replenishment of gas brings the galaxy back to a new circle of compaction. Supporting observational evidence includes that passive galaxies start to be abundant when the central stellar density reaches a threshold value (Fang et al. 2013; Woo et al. 2015; Tacchella et al. 2016; Mosleh et al. 2017; Whitaker et al. 2017); compact star-forming and compact passive galaxies have similar mass, kinematics, and morphology (Barro et al. 2013, 2014, 2017; Bruce et al. 2014; Nelson et al. 2014; Williams et al. 2014); and galaxies quench inside out (Tacchella et al. 2015; González Delgado et al. 2016; Barro et al. 2017; Belfiore et al. 2017; Brennan et al. 2017; Ellison et al. 2018).

For galaxies to stay on the SFMS, their star formation needs to be sustained by gas accretion (Kennicutt 1998; Putman 2017). The accreted gas needs to go through a whole process of cooling out of the circumgalactic medium (CGM, White \& Frenk 1991), infalling and settling into a dynamically and thermally cool atomic hydrogen (H I) disk, and further cooling and condensing into molecular $\left(\mathrm{H}_{2}\right)$ clouds (Krumholz 2012) in the inner disks, before finally fueling the star formation (Bigiel et al. 2008; Schruba et al. 2011). Thus, H I is a necessary intermediate phase for the fueling (sustaining) of star formation. In the more distant universe, the phase of $\mathrm{H} \mathrm{I}$ might be less important, for the neutral gas seems to be dominated by the molecular phase (Tacconi et al. 2013, 2018; but see also, e.g., Cortese et al. 2017; Decarli et al. 2019). But in the local universe, the conversion of $\mathrm{HI}$ to $\mathrm{H}_{2}$ is much less efficient, and $\mathrm{HI}$ is a significant component of the neutral gas ( $\sim 80 \%$ globally and 50\% in the stellar disks for star-forming galaxies; Catinella et al. 2018 and this paper). Hence, the $\mathrm{H} \mathrm{I}$ abundance and its connection to the $\mathrm{H}_{2}$ phase are important details to quantify observationally in order to constrain models of galaxy evolution.

At present, a most efficient way to directly observe neutral hydrogen gas for a statistically significantly large sample of galaxies is through single-dish radio telescopes (Saintonge et al. 2017; Catinella et al. 2018; Haynes et al. 2018). The integrated mass and velocity widths of the neutral gas obtained from single-dish surveys has greatly advanced our understanding of galaxy population and evolution. The $\mathrm{HI}$ and $\mathrm{H}_{2}$ mass fractions (gas mass over the stellar mass) are found to be 
correlated with the color, specific SFR, and effective stellar mass surface density of galaxies (Catinella et al. 2010, 2018; Saintonge et al. 2011, 2017; Tacconi et al. 2013). A high H I richness is also related to newly formed outer disks with low gas-phase metallicities (Moran et al. 2010, 2012; Carton et al. 2015) and an excess of young stars (Wang et al. 2011), a significant fraction of which are formed in bursts (Huang et al. 2013). H I-rich galaxies further tend to be in environments of low local densities and low dark matter halo masses (Fabello et al. 2012; Catinella et al. 2013; Hess \& Wilcots 2013; Brown et al. 2017). The depletion time of $\mathrm{HI}\left(M_{\mathrm{HI}}\right.$,tot $\left./ \mathrm{SFR}\right)$ is typically $3 \mathrm{Gyr}$ for different types of massive galaxies (Schiminovich et al. 2010), but can be close to the Hubble time for low-mass and low-SFR galaxies (Saintonge et al. 2017). The star-forming efficiency of $\mathrm{H}_{2}$ (characterized as $\mathrm{SFR} / M_{\mathrm{H}_{2}}$ ) is strongly correlated with the specific SFR (sSFR) and stellar surface density of galaxies (Saintonge et al. 2011, 2012; Tacconi et al. 2013; Huang \& Kauffmann 2014, 2015; Genzel et al. 2015). Studies of the distribution of galaxies in SFR $-M_{*}$ space found that the $\mathrm{H}$ I and $\mathrm{H}_{2}$ mass fractions are not only correlated with the extent of the SFR deviating from the SFMS, but also anticorrelated with the stellar mass along the SFMS (Saintonge et al. 2016). The former correlation is strengthened when the galaxies have high central stellar compactness (Wang et al. 2018).

However, the lack of spatial information has become a major limitation to linking the observed neutral gas properties to physical processes. Specifically, H I is typically more radially extended than the stellar disk in star-forming galaxies (Swaters et al. 2002; Wang et al. 2013), and the high angular momentum $\mathrm{HI}$ in the outer region cannot effectively feed the star formation, which is largely concentrated in the stellar disks (Bigiel et al. 2010; Yim \& van der Hulst 2016; Wang et al. 2017; Y1ldiz et al. 2017). In the future, the Square Kilometer Array (SKA) and its pathfinders will provide high-resolution H I images for a large area of the sky (de Blok et al. 2015; Staveley-Smith \& Oosterloo 2015) and will finally solve this problem. But before that, we already have the knowledge to roughly estimate the spatial distribution of $\mathrm{HI}$ gas from the integrated H I data, at least for late-type disk galaxies. The radial distributions of $\mathrm{H}$ I are self-similar in the outer regions of late-type galaxies when normalized to a characteristic radius $R_{\mathrm{H} \text { I }}$, defined as the semimajor axis of the $1 M_{\odot} \mathrm{pc}^{-2}$ isophote (Swaters et al. 2002; Wang et al. 2014, 2016, W16 hereafter). Partly due to this similarity, there is a tight linear relation between the H I mass $M_{\mathrm{H} \text { I,tot }}$ and $R_{\mathrm{H} \mathrm{I}}$, with a scatter of only 0.06 dex for estimates of $R_{\mathrm{H} \mathrm{I}}$ based on the relation (Broeils \& Rhee 1997; Verheijen \& Sancisi 2001; Swaters et al. 2002; Noordermeer et al. 2005; Wang et al. 2014, 2016; Martinsson et al. 2016). Combining these two facts, it is possible to guess / predict the outer part of the $\mathrm{H}$ I surface density radial profile of late-type galaxies given the H I mass, and hence divide the integrated H I mass into inner and outer parts. In this paper, we explore the feasibility and application of such a method. In particular, we study how the predicted H I located within the inner stellar disks will alter or reinforce our established view of $\mathrm{H}$ I fueling the star formation of star-forming galaxies.

The paper is organized as follows. We introduce the main sample selected from xGASS and xCOLD GASS, and a validation sample selected from $\mathrm{H}$ I interferometric surveys of nearby galaxies in Section 2. In Section 3, we describe the method of predicting the HI mass within the optical radius from the total H I mass, and use the validation sample to justify and calibrate the method. In Section 4, we apply the method to the main sample and analyze how H I masses and densities within the optical radius vary in the space of SFR versus $M_{*}$ of galaxies. We discuss the results in Section 5. We adopt a $\Lambda$ CDM cosmology with $\Omega_{m}=0.3, \Omega_{\lambda}=0.7$, and $h=0.7$. The initial mass function of Chabrier (2003) has been assumed for stellar mass and SFR estimates.

\section{Data \\ 2.1. The Main Sample}

This study is based on the xGASS (extended GALEX Arecibo SDSS Survey) representative sample (Catinella et al. 2018) of 1179 galaxies selected by stellar mass $\left(M_{*}>10^{9} M_{\odot}\right)$ and redshift $\left(0.01<z<0.02\right.$ for $M_{*}<10^{10} M_{\odot}$ and $0.025<$ $z<0.05$ for $\left.M_{*}>10^{10} M_{\odot}\right)$. The single-dish H I data were obtained with the Arecibo telescope, mostly in the GASS (Catinella et al. 2010, 2013) and GASS-low (Catinella et al. 2018) surveys, and complemented by data from ALFALFA (Giovanelli et al. 2005; Haynes et al. 2011) and the Cornell H I digital archive (Springob et al. 2005). Flags were provided for each H I spectrum to indicate the detection quality and possible confusion. Unless specifically noted, we do not account for the helium in the $\mathrm{HI}$ and $\mathrm{H}_{2}$ data used in this paper.

Additional multiwavelength information was collected from the public databases. Spectroscopic and photometric measurements are taken from SDSS-DR7 (Abazajian et al. 2009), including the redshift, the radii $r_{50}$ and $r_{90}$ (radius that enclose $50 \%$ and $90 \%$ of the total flux, respectively), and the radial distribution of surface brightness in the optical bands $u, g, r, i$, and $z$. We derive the average stellar surface densities within the central $1 \mathrm{kpc}$ through interpolating the radial profiles of surface brightness in the $g$ and $r$ bands, and converting the $r$-band surface brightness to stellar mass surface densities with a $(g-r)$-dependent $M_{*}$-to-light ratio from Zibetti et al. (2009). Additional estimates of galactic properties are taken from the MPA/JHU catalog (Kauffmann et al. 2003), including stellar mass $M_{*}$, for all galaxies and gas-phase metallicity $\mathrm{O} / \mathrm{H}$ for galaxies with strong emission lines.

SFRs were estimated based on the combination of NUV (from GALEX; Morrissey et al. 2007) and MIR (from WISE; Wright et al. 2010) luminosities (Janowiecki et al. 2017). There are 532 galaxies from xGASS followed up by the IRAM $30 \mathrm{~m}$ telescope to obtain the $\mathrm{CO}(1-0)$ emission line fluxes (xCOLD GASS, Saintonge et al. 2011, 2017). The conversion factor $\alpha_{\mathrm{CO}}$ was derived based on the metallicity and offset from the SFMS (Accurso et al. 2017), to convert the $\mathrm{CO}(1-0)$ fluxes to the $\mathrm{H}_{2}$ masses.

We select the disk-like, H I-detected galaxies from the xGASS representative sample by requiring $r$-band light concentration $r_{90} / r_{50}<2.7$, reasonable $\mathrm{HI}$ detection quality $\left(H I F L A G=1\right.$ or $\left.2^{8}\right)$, and no significant $\mathrm{H}$ I confusion $($ HIconfflag $=0)$. The selection on $r_{90} / r_{50}$ is to select (latetype) disk-like galaxies that have self-similar radial distributions of $\mathrm{HI}$ in the outer regions, and no significant stellar bulges which may affect the partition of neutral hydrogen into atomic and molecular phases in the inner regions. These properties are the basis of the method we are going to use to estimate the H I mass within the optical disks (Section 3). The

\footnotetext{
8 If we remove the HI_FLAG $=2$ marginal detections, the trends presented in this paper do not change.
} 
selection results in an xGASS disk sample of 447 galaxies. Among them, 179 have CO detections from xCOLD GASS, which make up the main sample of this paper.

\subsection{The Validation Sample}

We estimate the H I mass within the optical $r_{90}$ (rband) of galaxies, based on the integrated $\mathrm{H}$ I mass $\left(M_{\mathrm{H}} \mathrm{Itot}\right)$ and optical photometric measurements. We will calibrate and test the methods against a validation sample of nearby galaxies which have well-resolved and sensitive H I images (naturally weighted).

The validation sample (VS) includes galaxies from THINGS (Walter et al. 2008), WHISP-Sc (Swaters et al. 2002), and LVHIS (Koribalski et al. 2018). The stellar masses were calculated in Wang et al. (2017), based on Spitzer IRAC 3.6 and $4.5 \mu \mathrm{m}$ luminosities for THINGS, the $B$ - (from SIMBAD, Wenger et al. 2000) and $R$-band luminosities (from Swaters \& Balcells 2002) for WHISP-Sc, and the WISE 3.4 and $4.6 \mu \mathrm{m}$ luminosities for LVHIS. $r_{90}$ were measured from $3.6 \mu \mathrm{m}$ images for the THINGS galaxies (Leroy et al. 2008) and $3.4 \mu \mathrm{m}$ images for the LVHIS galaxies (Wang et al. 2017). Only $r_{80}$ is available for WHISP-Sc (Swaters \& Balcells 2002), so we approximate $r_{90}=1.1 r_{80}$ for these galaxies. SFRs of THINGS galaxies were derived by combining $24 \mu \mathrm{m}$ luminosities from SINGS (Kennicutt et al. 2003) and FUV luminosities from NGS (Gil de Paz et al. 2007). SFRs of LVHIS galaxies were estimated by combining the GALEX FUV and WISE $22 \mu \mathrm{m}$ luminosities in Wang et al. (2017). We use the same pipeline as Wang et al. (2017) to estimate the SFR for the WHISP-Sc galaxies.

We select the galaxies with $M_{*}>10^{9} M_{\odot}$ and $r_{90}>2.5 B_{\text {maj. }}$. The selection on $M_{*}$ is to ensure a similar $M_{*}$ range to the main sample. The selection on size is to ensure reliable measurements of the $\mathrm{H}$ I mass within $r_{90}$. We further select the galaxies with no significant missing-flux problems by requiring $D_{\mathrm{H} \mathrm{I}}<7^{\prime}$ for THINGS galaxies (so that the interferometric H I fluxes are consistent with the single-dish ones within a scatter of $\sim 10 \%$ after applying the selection criteria Walter et al. 2008), and $D_{\mathrm{HI}}<6 ! 7$ for WHISP-Sa galaxies (suggested by Swaters et al. 2002). VS includes 11, 29, and 10 galaxies from THINGS, WHISP-Sc, and LVHIS, respectively-in total 50 galaxies.

\section{Estimating H I Mass within the Optical $r_{90}$ of Disk-like Galaxies}

We present a method to estimate the H I mass within the optical $r_{90}, M_{\mathrm{H} \mathrm{I}, \text { in,pred }}$, based on $M_{\mathrm{H} \mathrm{I}, \text { tot }}$ and other optical properties. We compare $M_{\mathrm{HI} \text {,in,pred }}$ with the real measurements $M_{\mathrm{H} \mathrm{I} \text {,in }}$ to assess the method. The method discussed below is independent of the selected radius $r_{90}$, which can be replaced by other types of radius (e.g., $r_{25}$ where the optical band isophotes reach a surface brightness of $25 \mathrm{mag} \operatorname{arcsec}^{-2}$ ) in future applications.

\subsection{Method: Median $\Sigma_{H I}$ Observed for Galaxies}

This method makes use of two observational facts: (1) galaxies lie on a remarkably tight $D_{\mathrm{HI}}-M_{\mathrm{HI} \text { Itot }}$ relation (Swaters et al. 2002, W16), and (2) the $\Sigma_{\mathrm{HI}}$ profiles of late-type galaxies show homogeneous shapes in the outer regions, when the radius is normalized to $R_{\mathrm{HI}}\left(0.5 D_{\mathrm{HI}}\right.$,

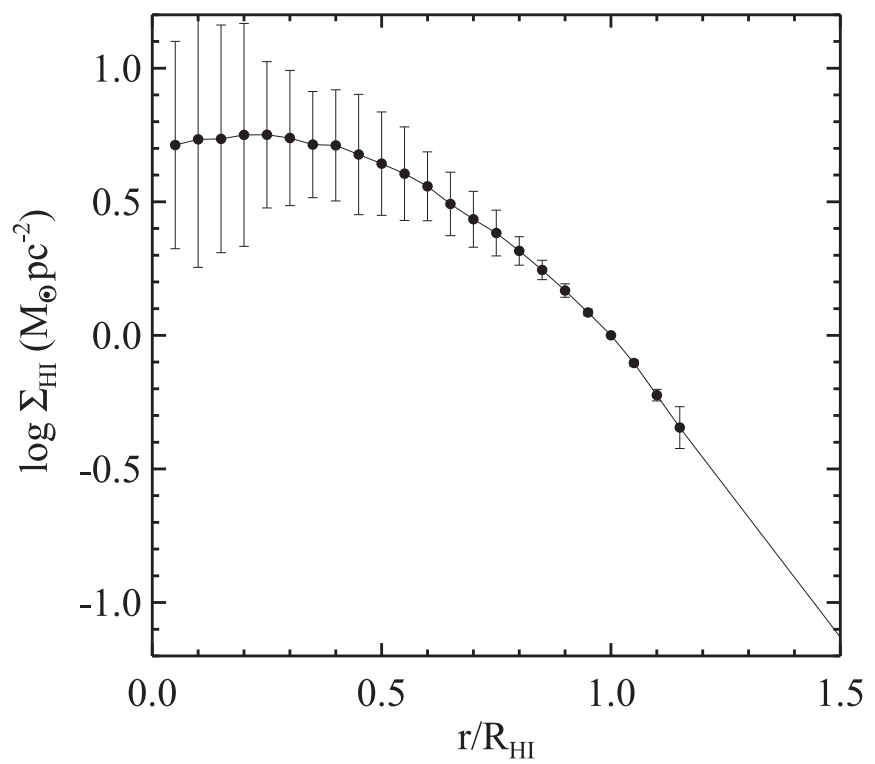

Figure 1. The W16 $\Sigma_{\mathrm{H}}$ profile, obtained as the median of the $\Sigma_{\mathrm{H}}$ profiles of 168 spiral and dwarf galaxies derived in W16. The directly derived profile extends to $1.15 R_{\mathrm{HI}}$ and has been extrapolated to $1.5 R_{\mathrm{H} \mathrm{I}}$.

Wang et al. 2014, W16). We take the median HI surface density profile from $\mathrm{W} 16$, which has the radius normalized to $R_{\mathrm{HI}}$, and was derived from a sample of 168 nearby spiral and dwarf galaxies, which have $R_{\mathrm{HI}}>3 B_{\text {maj }}$, where $B_{\text {maj }}$ is the major axis of the synthesis beam. The original median profile extends to a maximum radius of $1.15 R_{\mathrm{H}}$; we extrapolate it out to $1.5 R_{\mathrm{HI}}$ assuming an exponential outer disk with a scale length of $0.2 R_{\mathrm{HI}}$ (Wang et al. 2014, W16). We refer to the extrapolated profile as the $\mathrm{W} 16 \Sigma_{\mathrm{H} \text { I }}$ profile hereafter and show it in Figure 1.

We describe the procedure of estimating $M_{\mathrm{H} \text { I,in, pred }}$ below and also demonstrate it in Figure 2.

For each given $M_{\mathrm{H} \text { I,tot }}$ of a galaxy, we estimate the radius $R_{\mathrm{HI}}$ based on the $D_{\mathrm{H} \mathrm{I}}-M_{\mathrm{HI} \text {,tot }}$ relation. Then, we estimate $M_{\mathrm{HI} \text {,out,pred, }}$, the $\mathrm{HI}$ mass between $r_{90}$ and $1.5 R_{\mathrm{HI}}$ using the following procedure:

1. When $1.5 R_{\mathrm{HI}}>r_{90}$, we scale the radius of the W16 $\Sigma_{\mathrm{H} \text { I }}$ profile by $R_{\mathrm{H}}$, and hence obtain a "predicted $\Sigma_{\mathrm{H} \mathrm{I}}$ profile" for the galaxy. Then we cumulate the predicted $\Sigma_{\mathrm{HI}}$ profile between $r_{90}$ and $1.5 R_{\mathrm{H} \mathrm{I}}$ to estimate $M_{\mathrm{H} \mathrm{I} \text {,out,pred. }}$

2. When $1.5 R_{\mathrm{H} \mathrm{I}}<r_{90}, M_{\mathrm{H} \mathrm{I} \text {,out,pred }}=0$.

Finally, $M_{\mathrm{H} \text { I,in,pred }}=M_{\mathrm{H} \mathrm{I}, \text { tot }}-M_{\mathrm{H} \mathrm{I}, \text { out,pred}}$.

\subsection{Justification of the Method}

We justify the performance of the method by quantifying the difference between the real and predicted amount of H I within $r_{90}, \log M_{\mathrm{H} \mathrm{I}, \text { in,pred }} / M_{\mathrm{H} \mathrm{I,in}}$ and $\Sigma_{\mathrm{H} \mathrm{I,in,pred}}-\Sigma_{\mathrm{H} \text { I,in }}$. We have subtracted 0.04 dex from the direct estimates of $M_{\mathrm{H} \mathrm{I} \text {,in,pred, }}$, to minimize the scatter and median offset from real measurements in VS. The -0.04 dex offset is likely due to wiggles in the $\Sigma_{\mathrm{H} \mathrm{I}}$ profile of individual galaxies, which are missed by the median $\Sigma_{\mathrm{HI}}$ profile of W16 and cause $M_{\mathrm{HI} \text { Iout,pred }}$ to underestimate $M_{\mathrm{H} \mathrm{I} \text {,out }}$. This correction will also be added when the method is applied to the main sample. In this sense, VS serves not only as an assessment sample, but also as a calibration sample for our method. 
1.
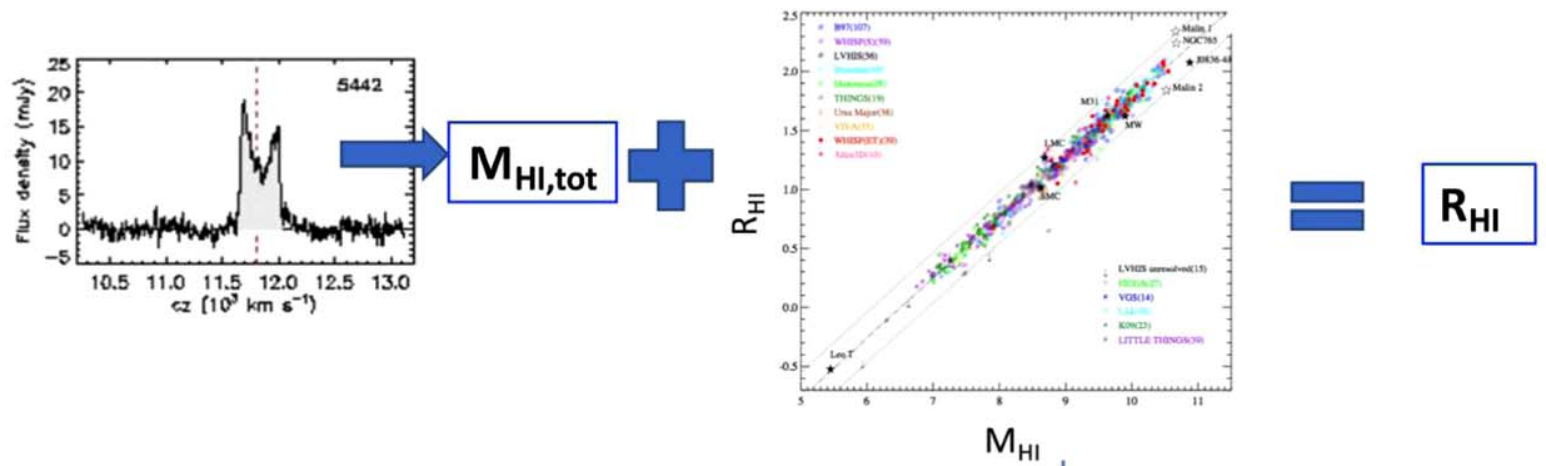

2.
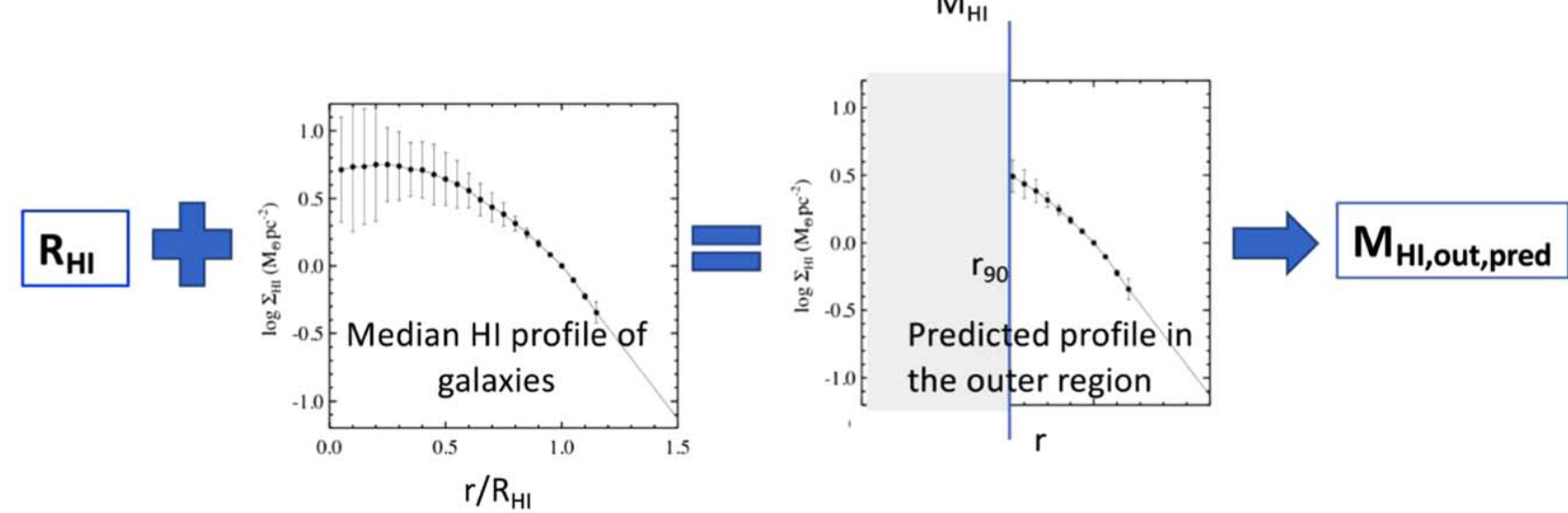

3.

\section{$M_{H I, \text { in, pred }}=M_{H 1, \text { tot }}-M_{H I, \text { out,pred }}$}

Figure 2. Three steps that estimate $M_{\mathrm{H} \text { I,in,pred }}$ based on the $M_{\mathrm{H} \text { I,tot }}$ of a galaxy.

Panel (a) of Figure 3 shows the good correlation and small offset between $M_{\mathrm{H} \text { I,in,pred }}$ and $M_{\mathrm{H} \text { I,in }}$. The scatter of $\log M_{\mathrm{HI} \text {,in,pred }} / M_{\mathrm{H} \text { I,in }}$ and $\Sigma_{\mathrm{HI} \text {,in,pred }}-\Sigma_{\mathrm{H} \text { I,in }}$ are $0.09 \operatorname{dex}$ and $0.6 M_{\odot} \mathrm{kpc}^{-2}{ }^{9}{ }^{\mathrm{VS}}$ has an average $\log M_{\mathrm{H} \text { I,in }} / M_{\odot}$ of $6.59 \pm 0.24 \mathrm{dex}$ and average $\Sigma_{\mathrm{H} \text { I,in }}$ of $4.2 \pm 2.2 M_{\odot} \mathrm{kpc}^{-2}$. Hence, the uncertainty of both estimates are 2-3 times less than the scatter of the real values within the sample.

Panels (b)-(e) of Figure 3 show that the scatter of $\log M_{\mathrm{H} \mathrm{I} \text {,in,pred }} / M_{\mathrm{H} \text { I,in }}$ for the VS galaxies does not significantly depend on $M_{*}$, sSFR, $M_{\mathrm{H} \mathrm{I}, \text { tot }} / M_{*}$, or $R_{\mathrm{HI}} / r_{90}$. The Pearson correlation coefficients suggest a weak anticorrelation of $\log M_{\mathrm{H} \mathrm{I} \text {,in,pred }} / M_{\mathrm{H} \mathrm{I} \text {,in }}$ with $M_{\mathrm{H} \mathrm{I} \text {,tot }} / M_{*}(\rho \sim 0.29)$. This weak anticorrelation does not significantly affect the interpretation of trends presented below in this paper, as long as the trends are

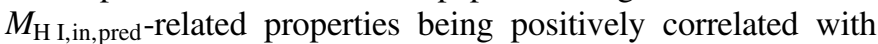
$M_{\mathrm{HI}, \text { tot }} / M_{*}$ (because if so, the real trend should be even stronger than observed).

Figure 4 shows that $\log M_{\mathrm{H} \text { I,in, pred }} / M_{\mathrm{H} \text { I,in }}$ do not show significant dependence on positions in the space of SFR versus $M_{*}$. It also shows that VS galaxies do not exactly overlap with the main sample galaxies in the space of SFR versus $M_{*}$. But we can see from both Figure 4 and the filled circles in Figure 3

\footnotetext{
9 We note that we have built a relatively small VS to match the $M_{*}$ range of the main sample. We also test the method with all the resolved late-type galaxies from W16, excluding the VIVA sample of galaxies in the Virgo cluster. We find $\log M_{\mathrm{HI} \text {,in,pred }} / M_{\mathrm{H} \text {, in }} \sim 0.02 \pm 0.11$ dex and $\Sigma_{\mathrm{H} \text { I, in,pred }}-\Sigma_{\mathrm{HI} \text {, in }} \sim 0.15 \pm 1.64 M_{\odot}$. The relatively large uncertainty in estimating $\Sigma_{\mathrm{H} \text { I, in }}$ is mainly due to the fact that the optical radius of dwarf irregular galaxies tends to be small and reach the non-exponential part of the W16 $\Sigma_{\mathrm{H} \text { I }}$ profile where the uncertainty is large.
}

that the overlapping and non-overlapping VS galaxies do not have significantly different uncertainties in $M_{\mathrm{HI} \text {,in,pred }}$ with respect to $M_{\mathrm{H} \text { I,in }}$.

We also find that $M_{\mathrm{H} \mathrm{I} \text {,in }}$ estimated with this observationmotivated method is as good as (or even better than) estimates produced by complex theoretical models (details in Appendix A). But this method requires much fewer inputs (only $M_{\mathrm{H} \text { I,tot }}$ and $r_{90}$ ) and relies on much fewer assumptions than those models.

These validations give us confidence that the method can be applied to the main sample to investigate the average $M_{\mathrm{H} \text { I,in }}$ and $\Sigma_{\mathrm{H} \text { I,in }}$ of galaxies along and around the SFMS. For simplicity, we refer to $M_{\mathrm{H} \mathrm{I}, \text { in,pred }}$ and $\Sigma_{\mathrm{H} \mathrm{I}, \text { in,pred }}$ as $M_{\mathrm{H} \mathrm{I} \text {,in }}$ and $\Sigma_{\mathrm{H} \text { I,in }}$ for the analysis of the main sample galaxies hereafter.

\subsection{Scaling Relation of $\mathrm{f}_{H \text { I, in }}$ in the Main Sample}

We apply our method of deriving $M_{\mathrm{H} \mathrm{I} \text {,in }}$ to the main sample of disk galaxies from xGASS. $\log M_{\mathrm{HI} \text {,in }} / M_{\mathrm{HI} \text {,tot }}$ ranges from -0.83 to -0.12 dex $(15 \%-76 \%$ in percentage, the 5 th and 95 th percentiles), with a width roughly $41 \%$ of the distribution width of $f_{\mathrm{H} \mathrm{I} \text {,tot }}\left(=M_{\mathrm{H} \mathrm{I}, \text { tot }} / M_{*}\right)$ and a median value of $-0.53 \mathrm{dex}$ (29\%). The relatively wide range and low median value of $M_{\mathrm{H} \mathrm{I}, \text { in }} / M_{\mathrm{H} \text { I,tot }}$ lend support to the necessity of considering the inner $\mathrm{HI}$ when studying the star-forming status of galaxies. We present in Figure 5 the scaling relations between $f_{\mathrm{H} \mathrm{I}, \text { in }}=M_{\mathrm{H} \mathrm{I}, \text { in }} / M_{*}$ and $M_{*}, \mu_{*}$ (the average stellar mass surface density with the $z$-band $\left.r_{50}\right), N U V-r$, and the specific $\mathrm{SFR}\left(\mathrm{sSFR}=\mathrm{SFR} / M_{*}\right)$. The trends are similar to the scaling relations of $f_{\mathrm{H} \text { I,tot }}$ (Catinella et al. 2018): galaxies tend to have 

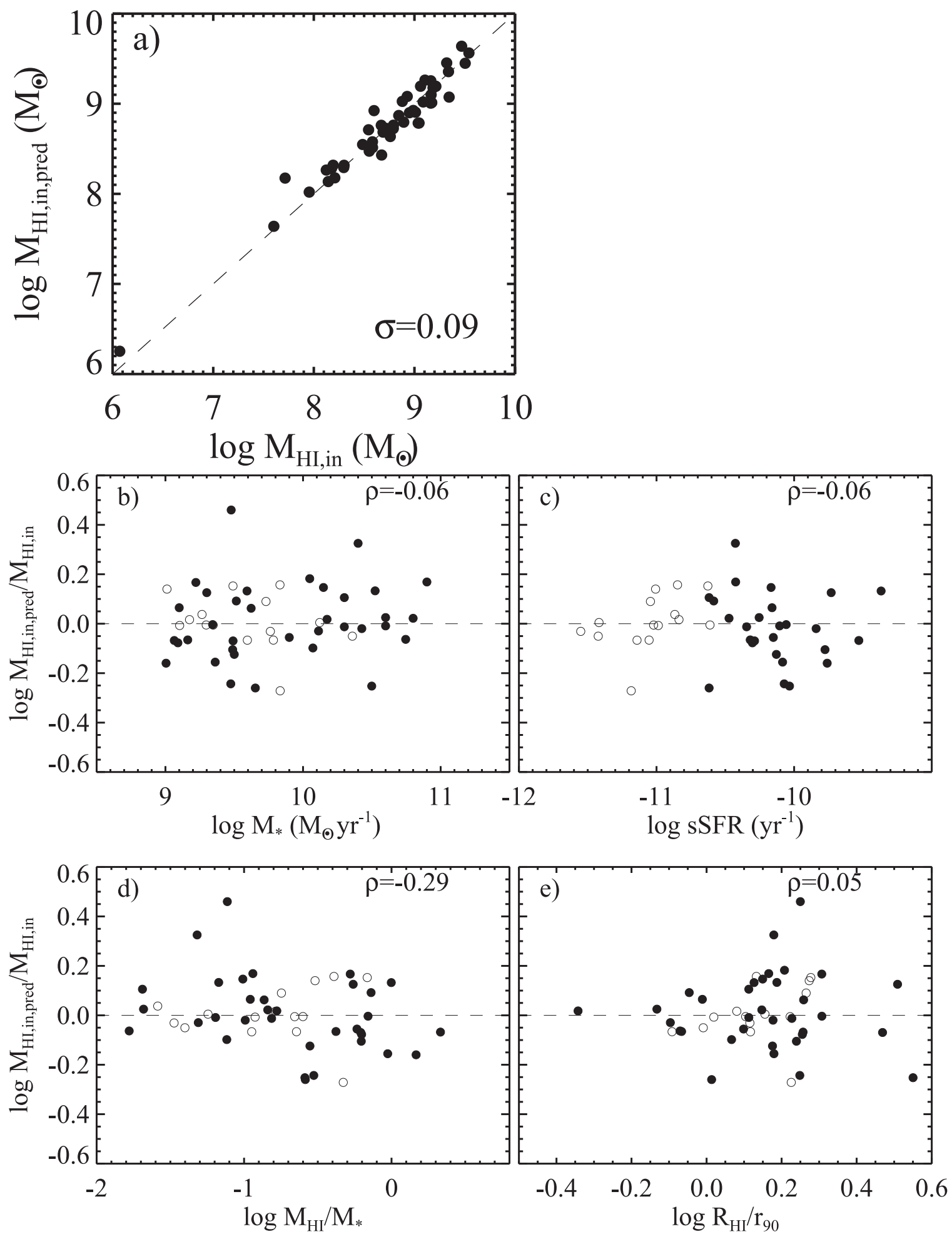

Figure 3. Comparison between the $M_{\mathrm{H} \text { I,in,pred }}$ and $M_{\mathrm{H} \text { I,in }}$ of the VS galaxies. In panel (a), the dashed line is the 1:1 line, and the scatter around the line is denoted in the corner. We also show the dependence of $\log M_{\mathrm{H} \text { I,in,pred }} / M_{\mathrm{H} \text { I,in }}$ on $M_{*}, \log \mathrm{sSFR}, M_{\mathrm{H} \text { I,tot }} / M_{*}$, and $R_{\mathrm{H}} / r_{90}$ in panels (b)-(e). In these panels, the dashed lines mark the position of $\log M_{\mathrm{H} \text { I,in,pred }} / M_{\mathrm{H} \text { I,in }}=0$, and the Pearson correlation coefficients are denoted at the corners. The open circles mark the VS galaxies that do not overlap with the main sample (squares in Figure 4).

higher $f_{\mathrm{H} \text {, in }}$ at lower $M_{*}$, lower $\mu_{*}$, bluer $N U V-r$, and higher sSFR. The slopes of the $f_{\mathrm{H} \text { I,tot }}$ and $f_{\mathrm{H} \mathrm{I}, \text { in }}$ relations differ most when the $x$-axis is $N U V-r$ or sSFR, because $f_{\mathrm{H} \text { I,tot }}$ and $f_{\mathrm{H} \text { I,in }}$ are close to each other when the $\mathrm{H}$ I disk shrinks into the stellar disks, i.e., when $N U V-r$ is red and SSFR is low. The scatter of the $f_{\mathrm{HI} \text {,in }}$ relations is always smaller than that of the corresponding $f_{\mathrm{H} \mathrm{I} \text {,tot }}$ relations, implying a closer link of $M_{\mathrm{H} \text { I,in }}$ with the stellar disks than $M_{\mathrm{H} \text { I,tot }}$. 


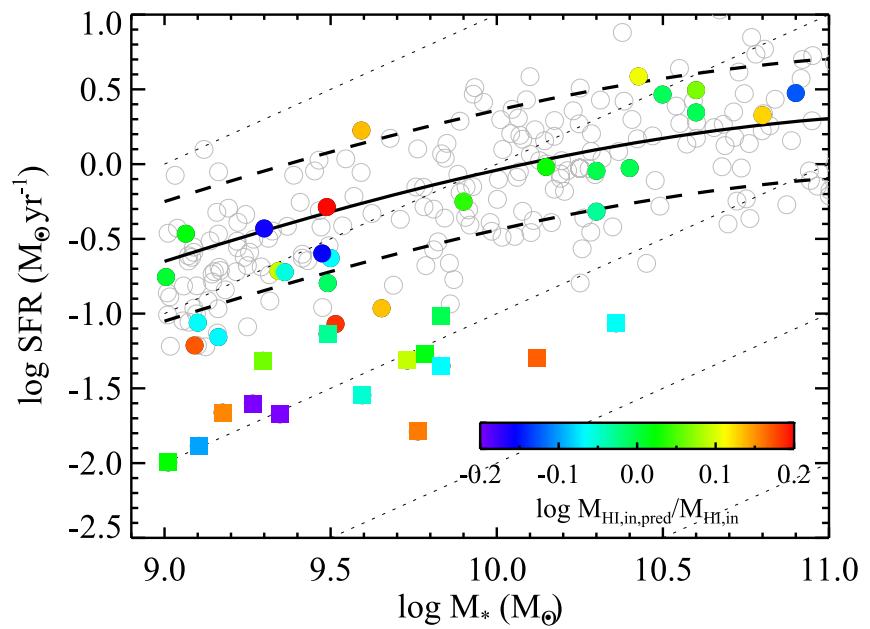

Figure 4. The distribution of VS galaxies in the space of SFR vs. $M_{*}$. The colors indicate $\log M_{\mathrm{HI} \text {,in,pred }} / M_{\mathrm{HI} \text {,in }}$ of VS galaxies (colored symbols). Colored squares mark the VS galaxies that do not overlap with the main sample (open, gray circles). The solid and dashed curves mark the mean position of the SFMS (Saintonge et al. 2016) and the 0.4 dex deviations. The dotted lines mark positions of constant sSFR, with separations of $1 \mathrm{dex}$.

\section{4. $M_{\mathrm{H} \text { I,in }}$ and $\Sigma_{\mathrm{H} \text { I,in }}$ of Galaxies in the Space of SFR versus M*}

This paper investigates the relation between the neutral gas reservoir of late-type galaxies and the bulk of the star formation which takes place in the stellar disk. We only consider the processes after the $\mathrm{HI}$ is accreted onto the disk from either the CGM or satellite galaxies. The HI in a HI-rich galaxy is radially more extended than the optical disk and needs to flow into the stellar disk where star formation can happen efficiently. The efficiency for the whole $\mathrm{H}$ I reservoir to become available for star formation within the stellar disk can be quantified as $M_{\mathrm{H} \text { I, in }} / M_{\mathrm{HI} \text {,tot }}$. The mass of this inner $\mathrm{HI}$ reservoir can be quantified as $M_{\mathrm{H} \text {, in }}$ or $f_{\mathrm{H} \text {, in }}=M_{\mathrm{H} \mathrm{I} \text {,in }} / M_{*}$. In addition, we use $\Sigma_{\mathrm{H} \text { I,in }}$, the average surface density of $\mathrm{H}$ I within $r_{90}$, as densities are physically more meaningful parameters to describe starforming activity than masses. The $\mathrm{HI}$ in the stellar disk will cool to form the molecular hydrogen and then stars. The efficiency of the former process can be quantified as $M_{\mathrm{H} \mathrm{I}, \text { in }} / M_{\mathrm{H}_{2}}$.

Finally, we use $M_{\mathrm{H}_{2}}+M_{\mathrm{H} \text {, in }}$ to indicate the total reservoir of material directly available for forming stars on the stellar disks. We define the depletion time for this immediate gas reservoir: $t_{\mathrm{dep}, \text { in }}=\left(M_{\mathrm{H}_{2}}+M_{\mathrm{H} \text { I,in }}\right) / \mathrm{SFR}$.

We investigate these neutral-gas-related parameters in the space of SFR versus $M_{*}$.

\subsection{Trends as a Function of $\mathrm{M}_{*}$ along the SFMS}

In Figure 6, we show how the neutral-gas-related parameters vary along the SFMS (equation from Saintonge et al. 2016) as a function of $M_{*}$ by averaging over disk galaxies (in total 132 galaxies from the main sample) which have their SFR within \pm 0.4 dex from the SFMS at fixed $M_{*}$.

The left panel shows the ratios of gas masses and SFR over $M_{*}$, which reflects the abundance of the gas reservoir in each state along the process of forming young stars. The middle panel shows the ratios of gas masses and SFR over gas masses, which reflects the efficiency of each step of the total H I reservoir being converted to stars. The $y$-axis of these two panels are displayed with the same width of 3.5 dex, so we can directly compare the slope of the observed trends (i.e., extent of variation of the parameters as a function of $M_{*}$ ) in these two panels. We can see that $f_{\mathrm{HI} \text {,tot }}, f_{\mathrm{H} \text { I,in }}, f_{\mathrm{H}_{2}}$, and sSFR show similarly strong decreases, while $M_{\mathrm{H} \text { I, in }} / M_{\mathrm{H} \mathrm{I} \text {,tot }}, M_{\mathrm{H}_{2}} / M_{\mathrm{H} \mathrm{I} \text {,in, }}$, and SFR $/ M_{\mathrm{H}_{2}}$ vary relatively weakly as a function of $M_{*}$.

Finally, the right panel shows that $\Sigma_{\mathrm{H} \text { I, in }}$ roughly decreases as a function of $M_{*}$.

\subsection{Trends as a Function of Deviation from the SFMS}

We now study the trend of neutral gas properties when disk galaxies of a given $M_{*}$ deviate from the SFMS, i.e., as a function of $\Delta \operatorname{logSFR}=\operatorname{logSFR} / \mathrm{SFR}_{\mathrm{SFMS}}$, where $\mathrm{SFR}_{\mathrm{SFMS}}$ is the mean SFR of star-forming galaxies on the SFMS at a given $M_{*}$.

The results are presented in Figure 7. The clearest trends we see are that, at a fixed $M_{*}$, higher SFR is on average related to higher $M_{\mathrm{HI} \text {,tot }}$ (panel (a)), higher $M_{\mathrm{H} \text { I,in }}$ (panel (b)), higher $f_{\mathrm{H} \text { I,tot }}$ (panel (c)), ${ }^{10}$ higher $f_{\mathrm{HI} \text {,in }}$ (panel (d)), higher $\Sigma_{\mathrm{HI} \text {,in }}$ (panel (c)), lower $M_{\mathrm{H} \mathrm{I} \text {,in }} / M_{\mathrm{H} \mathrm{I} \text {,tot }}(\operatorname{panel}(\mathrm{d}))$, and lower $t_{\mathrm{dep} \text {, in }}$ (panel (f)). Galaxies that have $\Delta \operatorname{logSFR}>0.4$ have the shortest $t_{\text {dep }} \lesssim 3 \times 10^{9} \mathrm{yr}$.

We also see that galaxies with higher SFR tend to have higher $M_{\mathrm{H}_{2}} / M_{\mathrm{H} \text { I,in }}$ when $M_{*}>10^{10} M_{\odot}$ (panel (e)). Such a trend is not observed in low-mass galaxies which have $M_{*}<10^{10} M_{\odot}$, but it is unclear whether it is affected by selection effects, as the low- $M_{*}$ and low-SFR galaxies are close to the CO detection limit of xCOLD GASS (Saintonge et al. 2017). Because $M_{\mathrm{H}_{2}} / M_{\mathrm{H} \text { I,in }}$ is also expected to be correlated with stellar surface density and gas-phase metallicity, one question is whether its enhancement in high-SFR and $M_{*}>10^{10} M_{\odot}$ galaxies (panel (e) of Figure 7 ) is due to a possibly systematic increase in stellar surface density or metallicity. Figure 8 confirms the strong and weak correlation

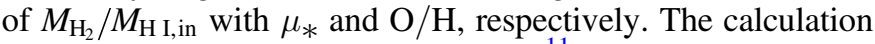
of the partial correlation coefficients ${ }^{11}$ suggests that when $M_{*}>10^{10} M_{\odot}$, the trend of $M_{\mathrm{H}_{2}} / M_{\mathrm{H} \text { I, in }}$ increasing with $\Delta \log$ SFR becomes stronger when the effect of $\mu_{*}$ or gas-phase metallicity $\mathrm{O} / \mathrm{H}$ is removed.

There might be a concern that the anticorrelation between $t_{\text {dep,in }}$ and SFR is due to SFR being present in both axes. The same applies to the relation between $f_{\mathrm{H} \text {,tot }}\left(f_{\mathrm{H} \mathrm{I} \text {,in }}\right)$ and $M_{*}$. Although this is true, the fact that the relations are not exactly linear shows that at least part of the correlation is not induced by plotting repeated quantities. Indeed, the relation between $t_{\text {dep,in }}$ and SFR has a negative slope, which indicates that SFR does not increase linearly as a function of $M_{\mathrm{gas}}$, but in a faster (superlinear) way. Similarly, the anticorrelation between $f_{\mathrm{H} \mathrm{I} \text {,tot }}$ $\left(f_{\mathrm{H} \text { I,in }}\right)$ and $M_{*}$ suggests that $M_{\mathrm{H} \mathrm{I}, \text { tot }}\left(M_{\mathrm{H} \mathrm{I}, \text { in }}\right)$ increases as a function of $M_{*}$ in a sublinear way.

Another possible concern is how random errors on the SFR and stellar masses affect these relations. To address this, we

\footnotetext{
${ }^{10}$ We notice that the slope for lines of constant $f_{\mathrm{H} \text {,tot }}$ in panel (a) looks smaller than that of $f_{\mathrm{H} \text { I, in }}$ in panel (d), which may give the false impression that for a given stellar mass, $f_{\mathrm{H} \text { I, in }}$ increases faster with SFR than $f_{\mathrm{HI} \text {,tot }}$. We point out that the false impression is due to the different color scales of the two panels, and panel (d) clearly shows the trend of $M_{\mathrm{H} \mathrm{I}, \text { in }} / M_{\mathrm{H} \mathrm{I} \text {,tot }}$ decreasing as a function of SFR.

${ }^{11}$ In order to calculate the partial correlation coefficient between parameters $A$ and $B$ with parameter $C$ controlled, one first derives the best linear fits of $A$ versus $C$ and $B$ versus $C$, and then the Pearson correlation coefficient between the offsets of both relations is calculated as the final partial correlation coefficient.
} 

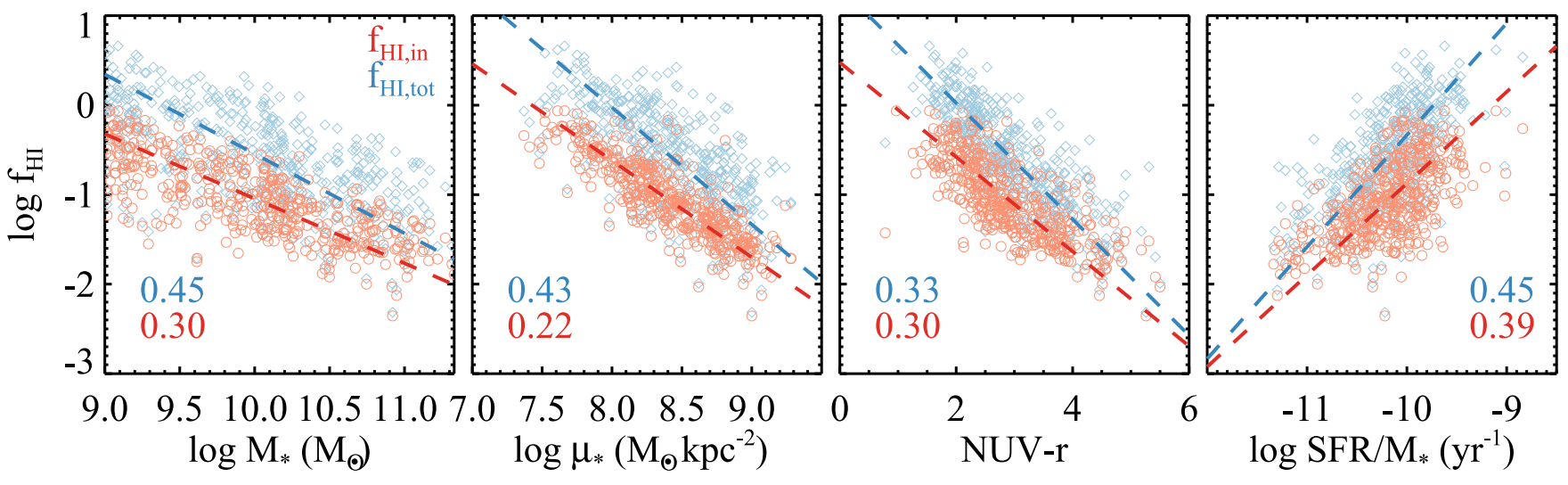

Figure 5. Scaling relations of $f_{\mathrm{H} \text { I,tot }}$ and $f_{\mathrm{H} \mathrm{I}, \text { in }}$. The dashed lines show the bisector linear fits. The scatters of the $f_{\mathrm{H} \mathrm{I}, \text { tot }}$ and $f_{\mathrm{H} \mathrm{I} \text {,in }}$ relations are shown in each panel. Galaxies from the xGASS disk sample are plotted.

calculate the error-corrected Pearson correlation coefficients for these relations, accounting for the estimated errors of SFR (typically $0.1 \mathrm{dex}$ ) and $M_{*}$ (typically $0.09 \mathrm{dex}$ ). We use the equation of Charles (2005): stellar disks quickly, but gas inflows do not seem to be very efficient (low $M_{\mathrm{H} \text { I,in }} / M_{\mathrm{H} \text { I,tot }}$ ) and thereby have built very extended H I disks (instead of concentrating the gas into the center and trigger starbursts) in these galaxies.

$$
r_{T x, T y}=\left(r_{x, y}-r_{E x, E y} * \sqrt{e_{x x} * e_{y y}}-r_{T x, E y} * \sqrt{r_{x x} * e_{y y}}-r_{E x, T y} * \sqrt{e_{x x} * r_{y y}}\right) / \sqrt{r_{x x} * r_{y y}},
$$

where $x$ and $y$ are measurements of two parameters, $E x$ and $E y$ are their errors, and $T x$ and $T y$ are their true values, so that $x=T x+E x$ and $y=T y+E y . r_{i, j}$ is the Pearson correlation coefficient between two quantities $i$ and $j, r_{i i}$ is the reliability of a measurement $i$, and $e_{i i}$ is the proportion of variance in measurement $i$ that is due to error. So, $r_{x, y}$ is the directly calculated Pearson correlation coefficient of the measurement, the correction terms with $r_{E x, E y}, r_{T x, E y}$ and $r_{E x, T y}$ correct for contributions from errors being correlated with each other or with the measurements, and the denominator of the equation corrects for the attenuation of the intrinsic correlation coefficients $\left(r_{T x, T y}\right)$ due to unreliable measurements. The error of each measurement ( $E x$ and $E y$ ) is simulated as a random value taken from a normal distribution with $\sigma$ equivalent to the measurement uncertainty $\left(\sigma_{x}\right.$ and $\left.\sigma_{y}\right)$. We further use the measurements $x$ and $y$ to approximate the intrinsic values $T x$ and $T y$ when calculating $r_{T x, E y}$ and $r_{T y, E x}$. We derive a corrected Pearson correlation coefficient of -0.52 for the relation between $t_{\mathrm{dep} \text {,in }}$ and SFR, -0.63 for the relation between $f_{\mathrm{H} \text { I,tot }}$ and $M_{*}$, and -0.73 for the relation between $f_{\mathrm{H} \text { I,in }}$ and $M_{*}$. These coefficients indicate strong correlations after taking into account the correlation of parameters with measurement errors. The approximation of approximate $T x(T y)$ with $x(y)$ while calculating $r_{T x, E y}\left(r_{T y, E x}\right)$ tends to overestimate the relevant correction terms and hence underestimate $r_{T x, T y}$, because the addition of $E x(E y)$ with respect to $T x(T y)$ is correlated with $E y(E x)$ for the parameter pairs considered here. So, the estimated error-corrected Pearson correlation coefficients $r_{T x, T y}$ are conservative and the correlation of these three parameter pairs are truly strong.

To summarize, the results suggest that SFR-enhanced galaxies tend to have built a large and dense $\mathrm{HI}$ reservoir within the stellar disks and achieved efficient atomic-tomolecular gas conversion in at least the $M_{*}>10^{10} M_{\odot}$ galaxies; they are likely to deplete the neutral gas on the

\subsection{Additional Dependence on Relative Stellar Compactness}

The average stellar surface density in the central $1 \mathrm{kpc}$ region, $\Sigma_{*, 1}$, quantifies the central stellar compactness (Cheung et al. 2012; Fang et al. 2013; Woo et al. 2015; Tacchella et al. 2016; Mosleh et al. 2017; Whitaker et al. 2017; Wang et al. 2018). Because the absolute compactness is correlated with $M_{*}$, it is useful to derive the mean relation between $M_{*}$ and $\Sigma_{*, 1}$, and to calculate $\Delta \Sigma_{*, 1}$, the deviation of $\Sigma_{*, 1}$ from the mean relation at a given $M_{*} . \Delta \Sigma_{*, 1}$ serves as an indicator of relative compactness at a given $M_{*}$. The relation between $\Delta \Sigma_{*, 1}$ and $\Delta_{\text {SFR }}$ has become a useful tool in investigations of the compaction scenario (Barro et al. 2017; Whitaker et al. 2017; Wang et al. 2018; Luo et al. 2019). Galaxies are observed to distribute in an "L" shape in the space of $\Delta \Sigma_{*, 1}$ and $\Delta_{\mathrm{SFR}}$, where high $\Delta_{\mathrm{SFR}}$ galaxies have a wide range of $\Delta \Sigma_{*, 1}$, but quenched galaxies almost all have high $\Delta \Sigma_{*, 1}$. The " $L$ " shape is consistent with the prediction of the compaction model, where galaxies first develop a compact stellar center with efficient star formation, before they cease star formation (Dekel \& Burkert 2014).

Panels (a) and (b) of Figure 9 show how SFR and $\Sigma_{\mathrm{H} \mathrm{I} \text {,in }}$ are distributed in the space of $\Sigma_{*, 1}$ versus $M_{*}$, respectively. At a given $M_{*}$, more compact galaxies tend to have lower SFR when $M_{*}>10^{10} M_{\odot}$; more compact galaxies also tend to have

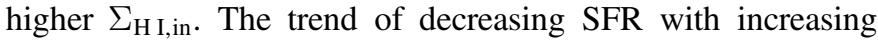
stellar compactness (at a given $M_{*}$ ) is consistent with Saintonge et al. (2016).

We then look into gas properties in the space of $\Delta \Sigma_{*, 1}$ versus $\Delta_{\mathrm{SFR}}$, which can be more conveniently compared to the compaction model. From panels (c) to (f), it is clear that our sample is selected against the really passive galaxies which typically have high $\Sigma_{*, 1}$ (the passive extension of the "L" shape is gray in the figure). Our study hence focuses on the fueling (toward compaction) but misses the quenching 

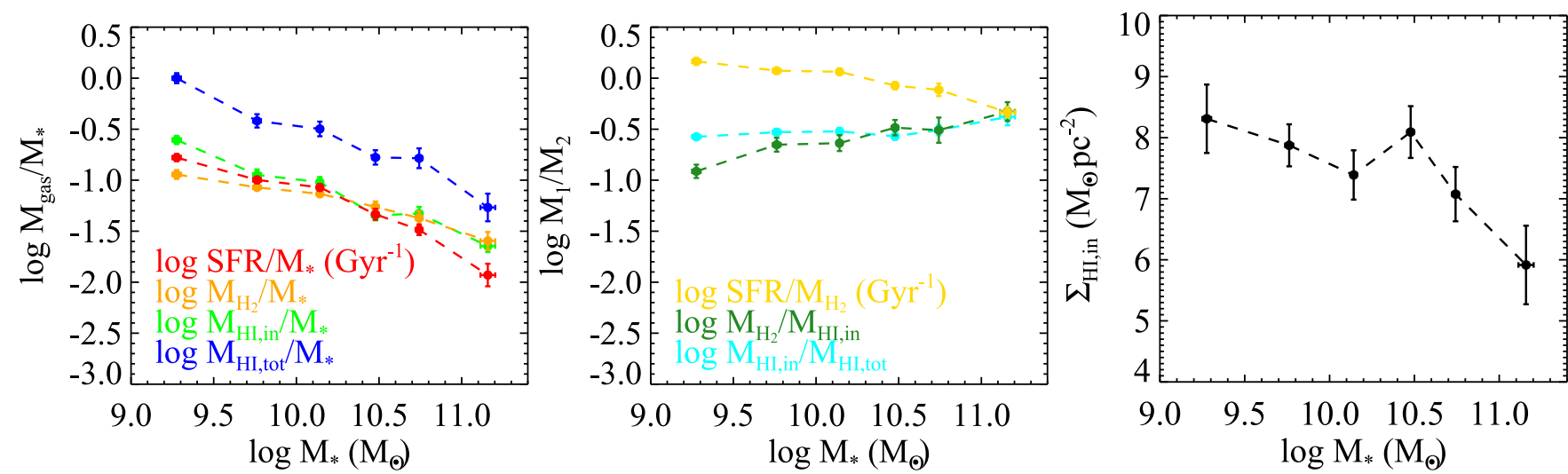

Figure 6. Properties of neutral gas along the SFMS for the main sample. Mean values and bootstrapped error bars are calculated \pm 0.4 dex from the SFMS (Saintonge et al. 2016) in each $M_{*}$ bin. Because the main purpose of the figure is to compare the dynamic range of parameters, we have added offsets of 9 and 8.5 dex in the left two panels to shift the SFR-related curves close to the other curves.

(following compaction) part of the compaction model (Tacchella et al. 2015).

Panel (c) of Figure 9 shows that less compact galaxies have higher $f_{\mathrm{H} \text { I,in }}$ than more compact galaxies. At low compactness, there is no significant correlation between $f_{\mathrm{H} \text { I,in }}$ and $\Delta \operatorname{logSFR}$. The lowest $f_{\mathrm{H} \text { I,in }}$ is found in galaxies with high compactness and low SFR enhancement.

$\Sigma_{\mathrm{H} \text { I,in }}$ increases (panel (d)) and $t_{\text {dep,in }}$ decrease (panel (e)) in the direction of $\Delta \log$ SFR and $\Delta \Sigma_{*, 1} \cdot M_{\mathrm{H}_{2}} / M_{\mathrm{H} \mathrm{I} \text {,in }}$ does not show a similarly strong trend to $\Sigma_{\mathrm{H} \text { I,in }}$ or $t_{\mathrm{dep}, \text { in }}$, but the highest values tend to be found where $\Delta \log$ SFR $>0$ and $\Delta \Sigma_{*, 1}>0$ (panel (c)). Hence, those compact, SFR-enhanced galaxies tend to build a denser neutral gas reservoir but deplete it more efficiently than less compact galaxies.

\section{Discussion}

We have developed a new method to estimate the H I mass $\left(M_{\mathrm{H} \mathrm{I}, \text { in }}\right)$ and surface densities $\left(\Sigma_{\mathrm{H} \text { I,in }}\right)$ within the optical $r_{90}$ for disk galaxies. $M_{\mathrm{H} \text { I,in }}$ serves as an intermediate state between the total $\mathrm{H} \mathrm{I}$ reservoir and the $\mathrm{H}_{2}$ disk. Although a large fraction of $\mathrm{H} \mathrm{I}$ in $\mathrm{H}$ I-rich galaxies lies beyond the stellar disk, $\mathrm{H}$ I within the stellar disk (compared to $\mathrm{H}_{2}$ ) is still likely the dominant reservoir for star formation (panel (e) of Figure 7), making the $t_{\text {dep,in }}(>3 \mathrm{Gyr})$ derived in this paper considerably longer than the $\mathrm{H}_{2}$ depletion time (Saintonge et al. 2017). We discuss below how the newly derived $M_{\mathrm{H} \text { I,in }}$ properties confirm or alter our previous understanding of galaxy evolution around the SFMS based on observations of global H I measurements. We emphasize that all results and discussions are limited to latetype disk galaxies. There are 348 and 142 galaxies from the xGASS disk and main samples respectively, which are identified as the central galaxy of groups (including isolated galaxies) in the catalog of Yang et al. (2007). We note that all $\mathrm{H}$ I-related trends presented in the paper do not change if we limit the analysis to late-type central galaxies.

\subsection{Trends Revealed with the Estimate of $\Sigma_{H I, \text { in }}$}

Because the real physical relation is between the surface densities of the SFR and the neutral gas, $\Sigma_{\mathrm{H} \text { I,in }}$ is a better tracer of SFR enhancement than $M_{\mathrm{H} \text { I,tot }}$ or $M_{\mathrm{H} \mathrm{I} \text {,in }}$ (panels (c) and (d) of Figure 9). The trend is about building a dense gas reservoir to begin and sustain highly efficient star formation and was not observationally demonstrated in the context of galaxy evolution for an $M_{*}$-selected and statistically significant sample of latetype galaxies.

We emphasize that the link between SFR and the inner H I appears similar to but different from the Kennicutt-Schmidt law of star formation (Kennicutt 1998), because H I serves not as the direct material (compared to the molecular gas) for star formation, but an intermediate state in the star formation fueling process. At an earlier stage of this process, an excess of warm $\left(10^{4} \mathrm{~K}\right)$ ionized gas in the CGM is observed around starforming galaxies with respect to passive galaxies in the local universe (Borthakur et al. 2015, 2016). It is also well accepted that star-forming galaxies tend to be globally $\mathrm{H} \mathrm{I}$ rich (Catinella et al. 2010, 2018). Our study further resolves the fueling process and finds that the $\mathrm{HI}$ within the stellar disks also builds up when galaxies tend to have high SFR. This trend is not so obvious before we quantify it, because $\mathrm{HI}$ is not the direct material for forming stars (Bigiel et al. 2008; Wang et al. 2017). It would be possible for one to speculate that at a given $M_{*}$, the galaxies with the highest SFR could have the lowest $M_{\mathrm{HI} \text {,in }}$, if $\mathrm{HI}$ in these galaxies were converted to $\mathrm{H}_{2}$ as efficiently as in the starbursting galaxies at high redshift (Tacconi et al. 2018). Our result suggests that this speculation is not true.

More compact galaxies on average may need higher inner H I surface densities to achieve the same extent of SFR enhancement (higher SFR surface densities due to smaller disk sizes) than less compact galaxies (panel (d) of Figure 9). These compact, star-formation-enhanced galaxies also deplete their neutral gas within the stellar disks more quickly than other galaxies (panels (c) and (d) of Figure 9). Their existence implies a possible evolutionary path for disk galaxies to develop a central bulge and cease star formation simultaneously, if gas replenishment is suppressed (e.g., if gas accretion is suppressed by a massive halo; Rees \& Ostriker 1977; Birnboim \& Dekel 2003; Kereš et al. 2005; van de Voort et al. 2011; Gabor \& Davé 2015, or lack of gas inflow due to weak disk instabilities, Noguchi 1998; Bournaud et al. 2007; Dekel et al. 2009; Cacciato et al. 2012). This is exactly the way the compaction model predicts how galaxies cease their star formation (Dekel \& Burkert 2014; Tacchella et al. 2015; Zolotov et al. 2015). These compact and SFR-enhanced galaxies are likely precursors of such an evolution (also see Ellison et al. 2018). 

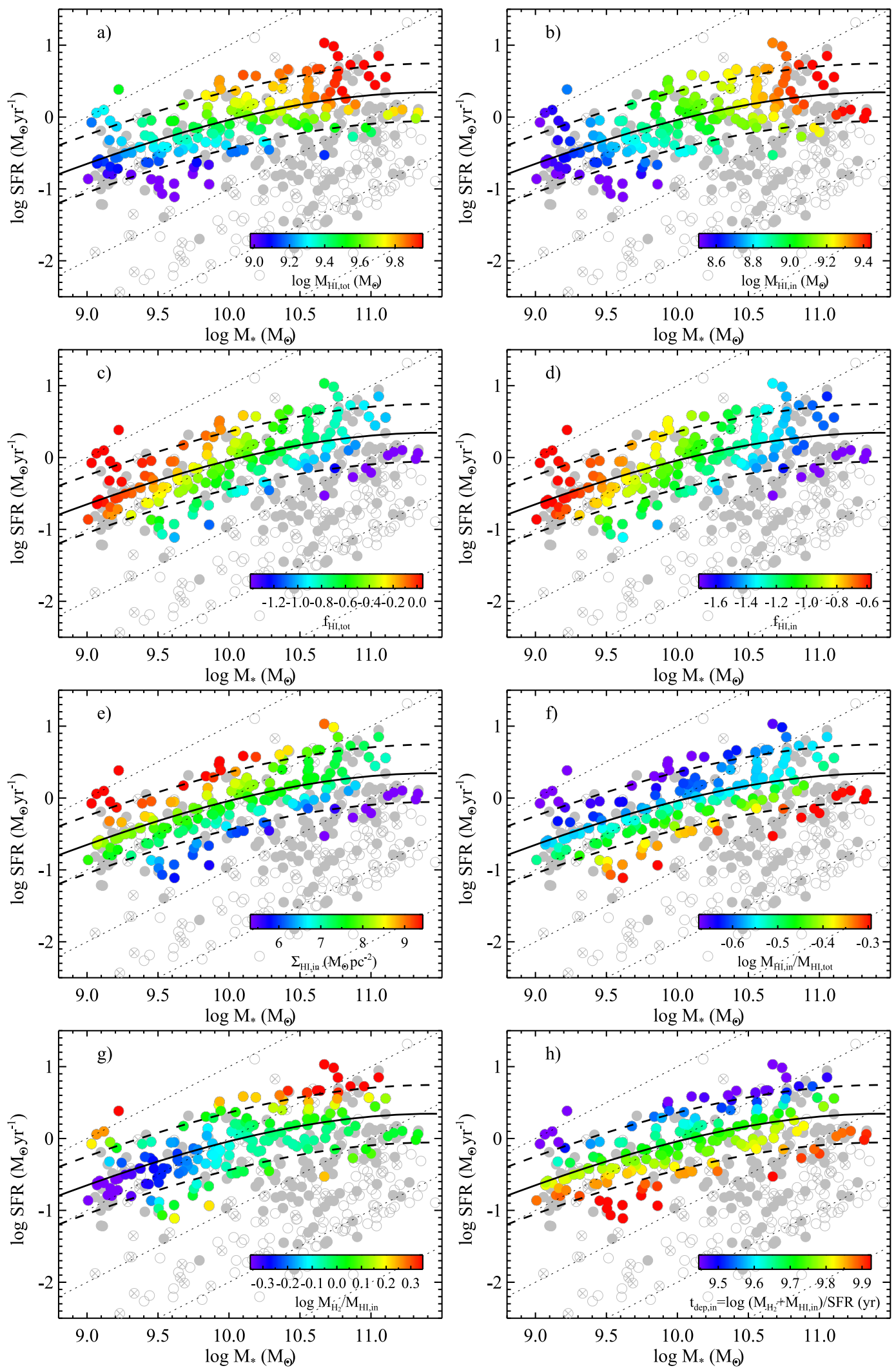

Figure 7. Properties of neutral gas in the space of SFR vs. $M_{*}$. The main sample galaxies are shown as color-coded dots, the xCOLD GASS bulge-dominated $\left(r_{90} /\right.$ $\left.r_{50}>2.7\right)$ galaxies which are detected in $\mathrm{H} \mathrm{I}$ and $\mathrm{CO}$ are shown as solid gray dots, and the xCOLD GASS disk/bulge-dominated galaxies which are undetected in $\mathrm{H}$ I or CO are shown as open gray circles with/without crosses. The color-coded quantities are LOESS-smoothed (Cappellari et al. 2013) to highlight the main trend. The color bars highlight the range between the 5th and 95th percentiles of the LOESS-smoothed distributions. The solid curve shows the mean position of the SFMS, and the dashed curves are vertically \pm 0.4 dex from solid curve. The diagonal dotted lines have slopes of unity and show positions of constant sSFR, with separations of $1 \mathrm{dex}$. 

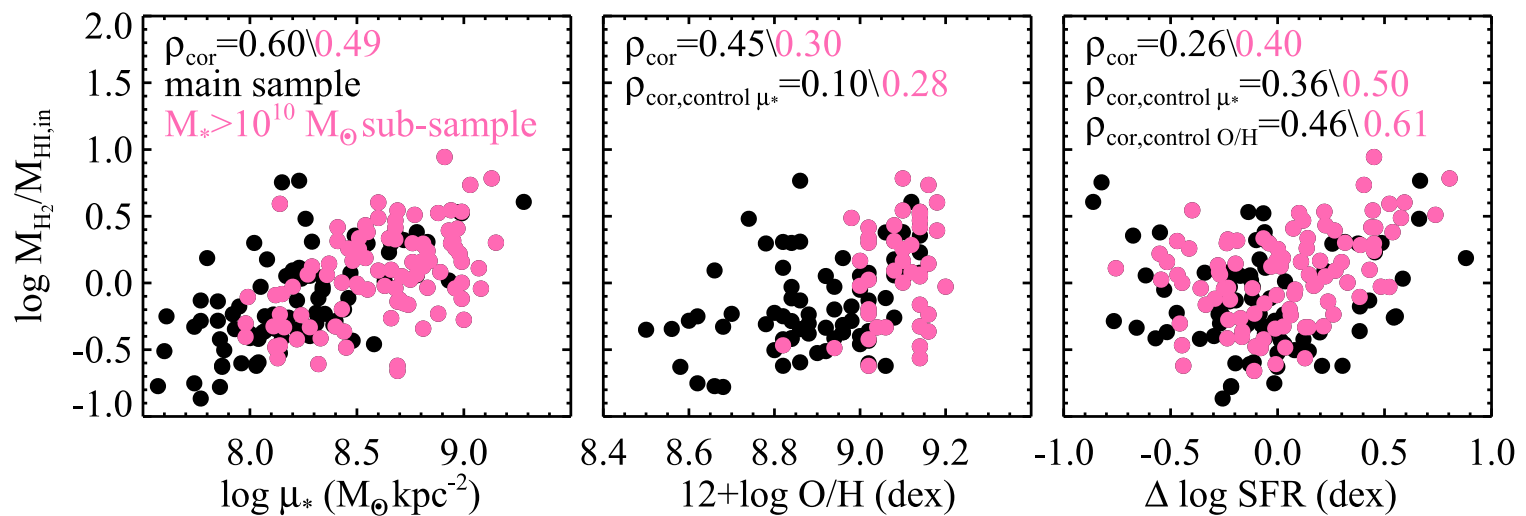

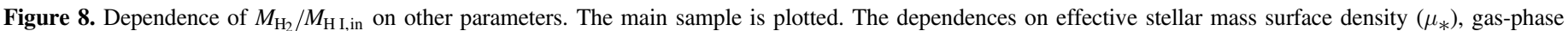

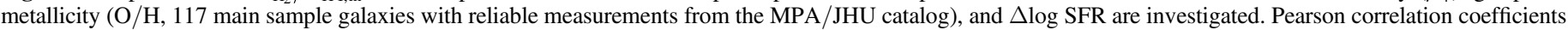

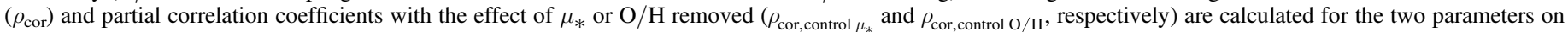
the $x$ - and $y$-axes of each panel. The data points and correlation coefficients of massive galaxies that have $M_{*}>10^{10} M_{\odot}$ are highlighted in pink.

The atomic-to-molecular conversion $\left(M_{\mathrm{H}_{2}} / M_{\mathrm{H} \mathrm{I}, \text { in }}\right)$ contributes to boosting the SFR, at least when $M_{*}>10^{10} M_{\odot}$ (panel (g) of Figure 7 and right panel of Figure 8). This effect was not observed with global measurements (Catinella et al. 2018), but is predicted in the compaction model (Dekel \& Burkert 2014; Tacchella et al. 2015; Zolotov et al. 2015). We can also see in Figure 10 that the global measurement $M_{\mathrm{H}_{2}} / M_{\mathrm{H} \text { I,tot }}$ does not increase monotonically with SFR at a given $M_{*}$, but reverse to show an increase when the galaxies are below the SFMS. As a result, the Pearson correlation coefficient between $M_{\mathrm{H}_{2}} / M_{\mathrm{H} \text { I,tot }}$ and $\Delta \log$ SFR is only $0.14\left(0.46\right.$ for the $M_{\mathrm{H}_{2}} / M_{\mathrm{H} \text { I, in }}$ versus $\Delta \log$ SFR relation), and the partial correlation coefficient with the effect of $\mu_{*}$ controlled is 0.28 ( 0.61 for the $M_{\mathrm{H}_{2}} / M_{\mathrm{H} \mathrm{I} \text {,in }}$ versus $\Delta \log$ SFR relation). This reversal in trend is likely due to the shrink of the $\mathrm{HI}$ disks instead of the enhanced conversion efficiency at low SFR.

Finally, a relatively low efficiency of gas inflows (lower $M_{\mathrm{H} \text { I,in }} / M_{\mathrm{H} \mathrm{I} \text {,tot }}$ for more star-forming galaxies) may be a major obstacle in fueling the SFR (panel (f) of Figure 7). Theoretically, under a CDM cosmological context, the largescale accreting gas has a high specific angular moment (Mo et al. 1998), while the star-formation-induced accretion of gas from the inner parts of the CGM (fountain gas) also tends to have high specific angular moment, due to a mixing with the high angular momentum CGM (Grand et al. 2019), and possibly also due to the suppression of low-angular momentum fountains with short dynamic times (Marasco et al. 2012). The gas with a high specific angular momentum tends to build an extended H I disk. We would expect much more vigorous star formation than observed if the massive $\mathrm{HI}$ in the outer disks of $\mathrm{H}$ I-rich galaxies could be efficiently driven to the center. The relatively low efficiency might be related to the fact that disk instabilities and tidally interacting frequencies are relatively low at low redshift compared to high redshift (Noguchi 1998; Bournaud et al. 2007; Dekel et al. 2009; Cacciato et al. 2012). It may also be related to the theoretically predicted selfregulation of low-redshift disks (Krumholz et al. 2018), where inflows are driven by disk instabilities (associated with low gas velocity dispersion and low Toomre $Q$ ), and a high inflow rate will result in high SFR and increased gas velocity dispersion (due to stellar feedback and gravitational heating of the inflow gas), which then suppress the disk instabilities (high Toomre $Q)$. Such a mechanism prevents strong inflows and maintains the extended $\mathrm{H}$ I disks in H I-rich galaxies. This feature was not described in classical compaction models (e.g., Dekel \& Burkert 2014; Tacchella et al. 2015; Zolotov et al. 2015) and may serve as a new constraint for these models in the local universe.

\subsection{Known Trends of $\mathrm{M}_{H \text { I,tot }}$ Confirmed by $\mathrm{M}_{H \text { I, in }}$}

One major feature of the SFMS is its slope being shallower than one. It was found that along the SFMS, $f_{\mathrm{H} \text { I,tot }}$ and $f_{\mathrm{H}_{2}}$ drop much faster than $M_{\mathrm{H}_{2}} / M_{\mathrm{H} \text { I,tot }}$ as a function of $M_{*}$ (Saintonge et al. 2016, 2017; Catinella et al. 2018). Hence, it is the shrinking of the gas reservoir, rather than a bottleneck in converting the atomic gas to the molecular gas, that plays a major role in the flattening of the SFMS. Our results (both $M_{\mathrm{H} \mathrm{I}, \text { in }} / M_{\mathrm{H} \mathrm{I} \text {,tot }}$ and $M_{\mathrm{H}_{2}} / M_{\mathrm{H} \text { I, in }}$ vary little with $M_{*}$ along the SFMS; middle panel of Figure 6) elaborate that when the SFMS flattens, the major bottleneck (of forming stars) is neither in driving $\mathrm{HI}$ inward to the stellar disks nor in converting $M_{\mathrm{H} \text {, in }}$ to $M_{\mathrm{H}_{2}}$ with the stellar disks. SFR/ $M_{*}, M_{\mathrm{H}_{2}}$, and $M_{\mathrm{H} \text { I,in }} / M_{*}$ drop fast, similarly to $M_{\mathrm{HI} \text {,tot }} / M_{*}$ along the SFMS (left panel of Figure 6), hence it is indeed likely (as concluded by Saintonge et al. 2016) that the global H I abundance (as the first step of fueling from $\mathrm{HI}$ to star formation) strongly regulates the slope of the SFMS.

The most star-forming galaxies rarely go far $(>0.4 \mathrm{dex}$ in SFR) above the SFMS. A short depletion time of the total neutral gas and molecular gas was found for the starbursting galaxies with respect to normal star-forming galaxies (Genzel et al. 2015; Silverman et al. 2015; Scoville et al. 2016; Saintonge et al. 2017). The trend is observationally related to the superlinear nature of the Kennicutt-Schmidt law of star formation (Kennicutt 1998). The more efficient depletion is taken as the major confinement for the upper envelope of the SFR $-M_{*}$ relation in the compaction model (Tacchella et al. 2015). We show that the trend is still true for disk galaxies when only the neutral gas with the stellar disks is considered (panel (h) of Figure 7).

Most of the star-forming galaxies have a scatter of \pm 0.4 dex in SFR around the SFMS. Previous observations found that $\Delta \log$ SFR at a given stellar mass is strongly set by $M_{\mathrm{H} \mathrm{I}, \text { tot }}$ and $M_{\mathrm{H}_{2}}$ (Whitaker et al. 2012; Tacchella et al. 2015; Saintonge et al. 2016, 2017; Catinella et al. 2018). The determining role 

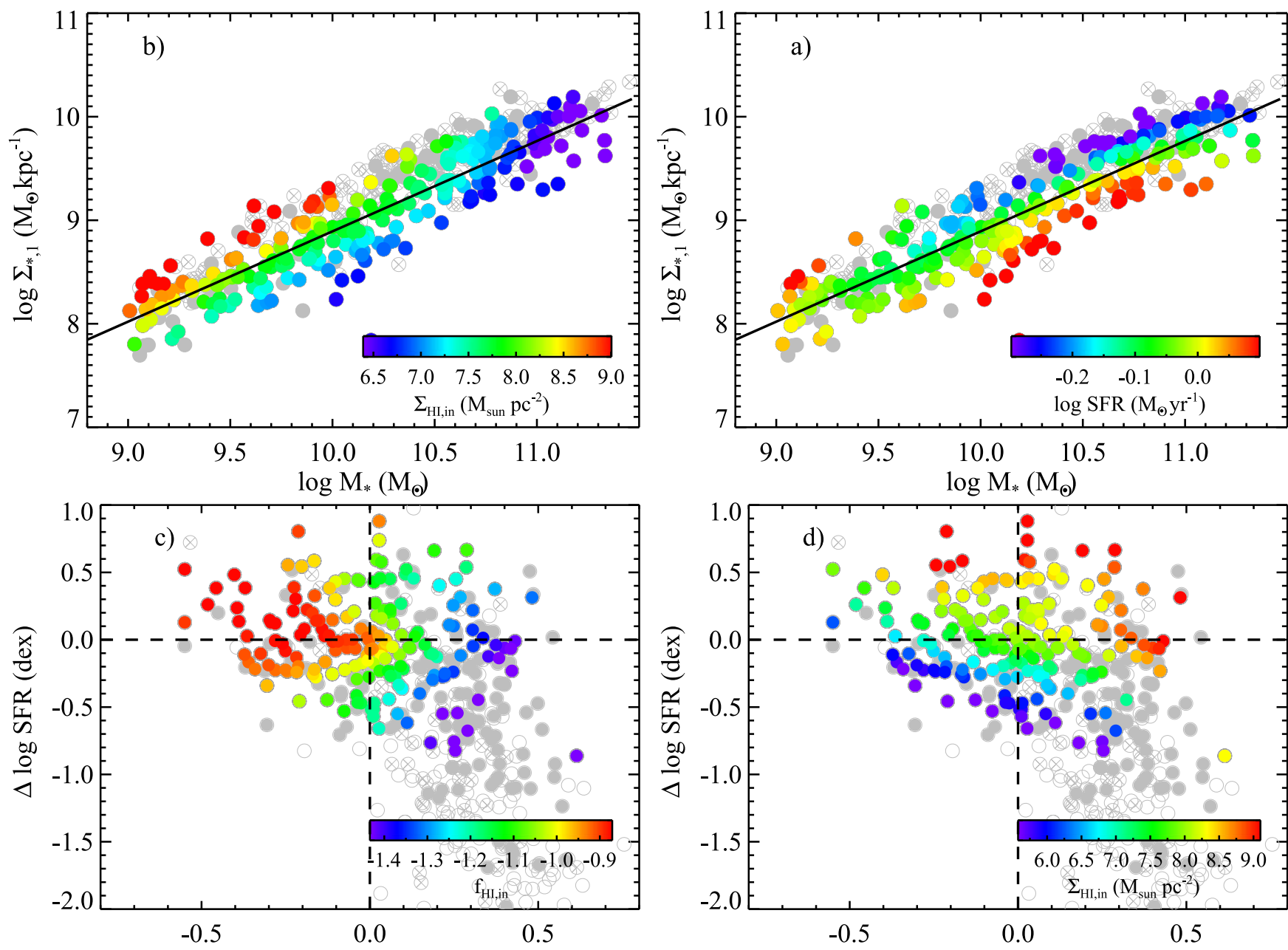

$\Delta \log \Sigma_{*, 1}(\mathrm{dex})$
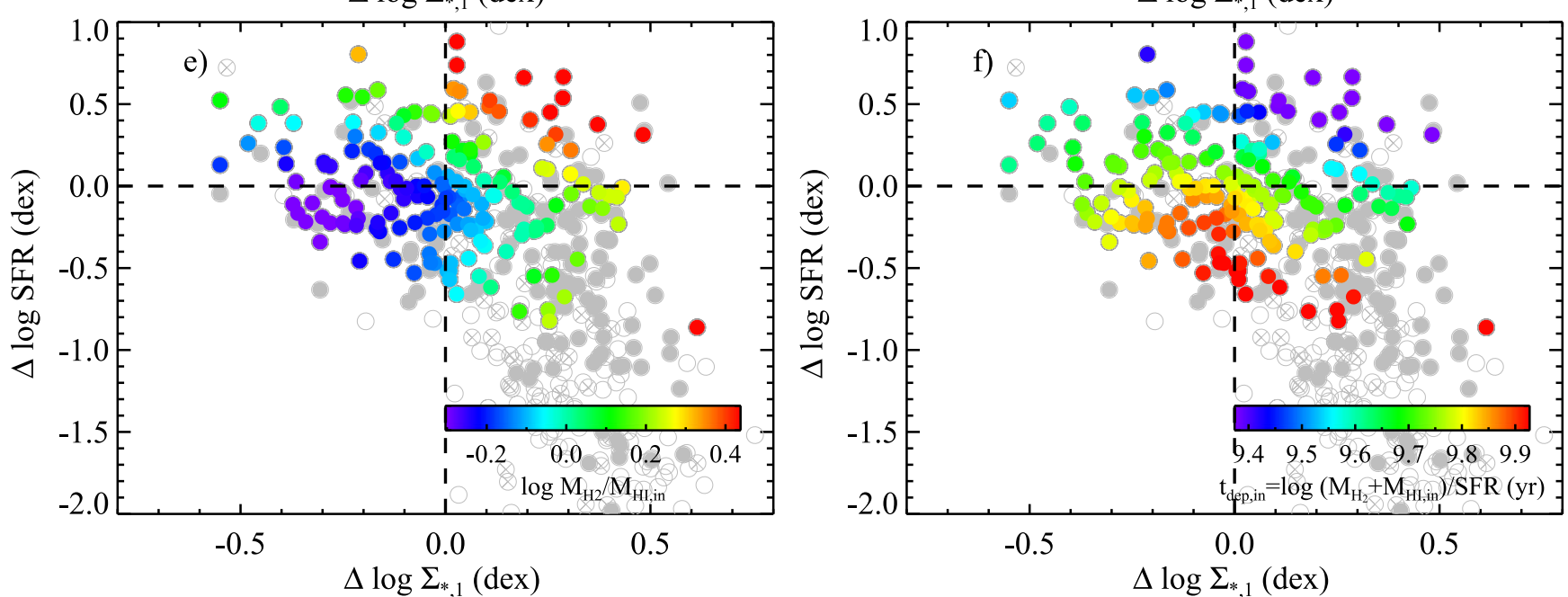

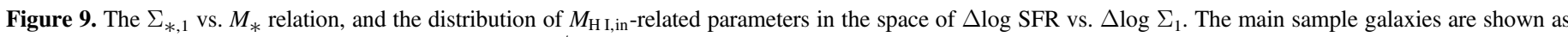

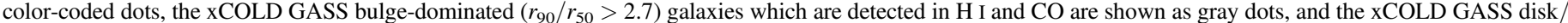

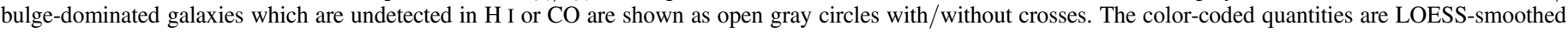
(Cappellari et al. 2013) to highlight the main trend.

of the cold gas reservoir on the star-forming status of galaxies was predicted by the compaction model (Tacchella et al. 2015). Our results confirm that on average the SFR has to increase with the reservoir of fueling material, globally as well as within the stellar disks for disk galaxies (panels (a)-(d) of Figure 7).

\subsection{Caveats and Future Perspective}

The main sample (on which most results are based) is very strongly biased toward the SFMS, as shown by the open circles with crosses in Figures 7 and 9. Therefore, our results strongly support the compaction scenario (see discussion in 


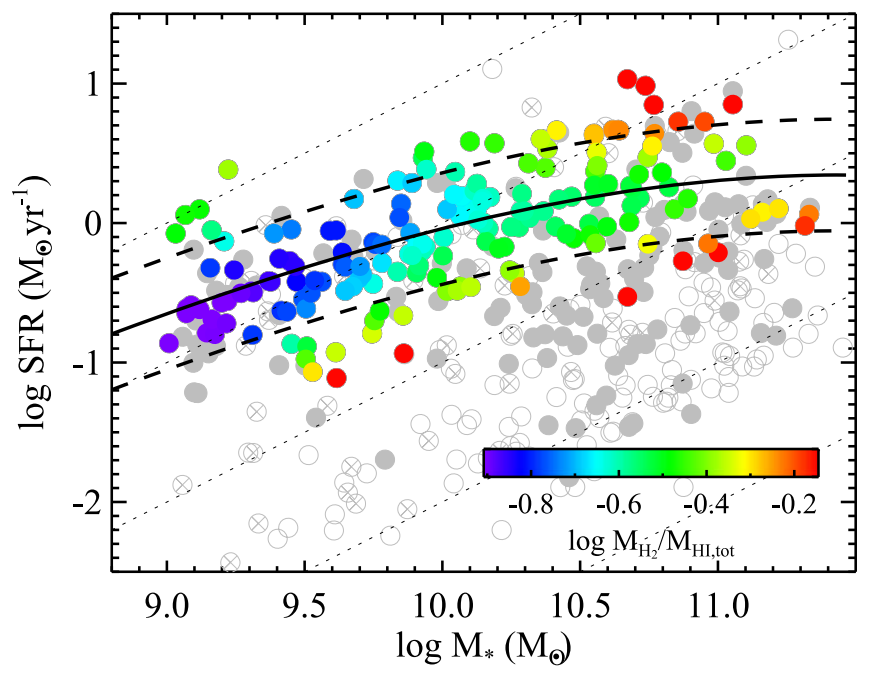

Figure 10. Distribution of $M_{\mathrm{H}_{2}} / M_{\mathrm{H} \text { I,tot }}$ in the space of SFR vs. $M_{*}$. Similar to Figure 7 , but the dots are color coded by $M_{\mathrm{H}_{2}} / M_{\mathrm{H} \text { I,tot }}$.

Section 5.1), but do not directly link to the model-predicted consequence that galaxies cease their star formation as a result of compaction (Dekel \& Burkert 2014; Tacchella et al. 2015; Zolotov et al. 2015). It is unclear which factors or mechanisms most strongly suppress SFR in the passive and massive disk galaxies, i.e., whether $f_{\mathrm{H} \text {,in }}$ and $\Sigma_{\mathrm{HI} \text {,in }}$ continuously drops (following the trends in panels (a), (c) and (d) of Figure 9), or $\mathrm{H}$ I for a given gas density forms too little molecular gas.

We need to keep in mind that predicted H I properties have deviations from the real ones. We caution the reader for unknown systematic dependences of the deviations, because the VS used to test our method is a relatively small sample with a complex selection. Processes that break the equilibrium state of galaxies, like tidal effects and episodic gas accretion, may affect the radial distribution of $\mathrm{HI}$. Hence, our discussion is limited to the average trend of disk-dominated galaxies where these effects are assumed to be normal. Moreover, $M_{\mathrm{H} \text { I,in }}$ and $\Sigma_{\mathrm{HI} \text {,in }}$ are estimated within the $r$-band $r_{90}$, which should enclose most but not exactly $90 \%$ of SFR and $\mathrm{H}_{2}$ gas. This mismatch adds uncertainty to the interpretation of our results, which may have a systematic dependence on the bulge-to-disk ratio of galaxies. We test the significance of this effect by replacing $r_{90}$ with $R_{25}$, the semimajor axis of the 25 mag $\operatorname{arcsec}^{-2}$ isophotes, which should be less dependent on the bulge prominence than $r_{90}$. We find that all of our trends remain (examples in Appendix B), though with a smaller amplitude due to the averaging of $\Sigma_{\mathrm{H} \text { I }}$ within a larger aperture than $r_{90}$. We hence conclude that the possible dependence of $r_{90}$ on bulge prominence does not significantly affect our major results and conclusion. Nevertheless, confirmation of our results will be needed in the future with real, spatially resolved HI data, which will be available when the new radio interferometric instruments finish their planned, large surveys of $\mathrm{HI}$ in nearby galaxies in the near future (ASKAPWALLABY, Apertif, etc.; de Blok et al. 2015; Staveley-Smith \& Oosterloo 2015).

The estimated $M_{\mathrm{HI}}$ in has promising applications in the moderate-redshift SKA and pathfinder H I surveys (DINGO, LADUMA, etc.; Meyer 2009; Holwerda et al. 2012), as well as the low-redshift ones (Staveley-Smith \& Oosterloo 2015; WALLABY), where $\mathrm{HI}$ in most of the galaxies will be unresolved. In addition to serving as an intermediate reservoir for star formation, it has the potential of improving $\mathrm{H}_{2}$ indicators. Due to the lack of large-sample millimeter surveys, $\mathrm{H}_{2}$ is often indicated by measurements of dust from infrared photometry or optical spectroscopy data (Brinchmann et al. 2013; Berta et al. 2016; Yesuf \& Ho 2019). Because dust seems to more closely trace the total neutral gas than $\mathrm{H}_{2}$ (Groves et al. 2015; Janowiecki et al. 2018) and the relations between dusts and neutral gas are different within and beyond the stellar disks (possibly due to the different gas-phase metallicities; Moran et al. 2012; Janowiecki et al. 2018), the estimated $M_{\mathrm{H} \text { I,in }}$ provide useful constraints on $M_{\mathrm{H}_{2}}$ estimators based on dust properties. Such a potential application will be investigated in a future paper.

\section{Conclusions}

The method presented offers a useful way to get more information out of global HI profiles for late-type (diskdominated) disk galaxies. This will be important for deep interferometric surveys such as DINGO (Meyer 2009) and LADUMA (Holwerda et al. 2012), and also single-dish FAST H I surveys ( $\mathrm{Li}$ et al. 2018; Zhang et al. 2019), as most detections will be unresolved. The method is able to characterize the average H I surface density within the stellar disk of late-type galaxies, where the gas is directly fueling the star formation. Exploring $\mathrm{H}$ I-related parameters in relation to the SFMS, we found that the spread along the SFMS is best characterized by this inner H I surface density (i.e., this parameter is the most discriminatory perpendicular to the SFMS, especially when considering the conversion of the $\mathrm{H}$ I to the molecular gas or fixing the central compactness of galaxies) among the H I-related parameters. So, for studying the spread of late-type galaxies in the SFMS, this is a quantity that one should focus on.

The trends found are generally consistent with the compaction model of galaxy evolution regulated by the balance between cold gas fueling and star formation depletion (Dekel \& Burkert 2014; Tacchella et al. 2015; Zolotov et al. 2015).

We gratefully thank Thijs van der Hulst for useful discussions. This work was supported by the National Science Foundation of China $(11721303,11991052)$ and the National Key R\&D Program of China (2016YFA0400702). Z.P. acknowledges the support from the National Natural Science Foundation of China (NSFC, grant No. 11703092). Parts of this research were supported by the Australian Research Council Centre of Excellence for All Sky Astrophysics in 3 Dimensions (ASTRO 3D), through project number CE170100013. J.W. further thanks support from the ASTRO 3D Science Visitor program at the ICRAR node.

\section{Appendix A \\ Comparing Our Method with that of Obreschkow et al. (2009) \\ A.1. Method O09: Model $\Sigma_{H I}$ Analytically Considering the $\mathrm{HI}-\mathrm{H}_{2}$ Conversion}

\section{A.1.1. Model 1: the Original Model of Obreschkow et al. (2009)}

Motivated by the following results, Obreschkow et al. (2009, O09 hereafter) proposed an analytical model for the radial distribution of $\Sigma_{\mathrm{HI}}$. 
1. Both the surface density of the total neutral gas $\left(\mathrm{H} \mathrm{I}+\mathrm{H}_{2}\right)$ and the gas conversion ratio $\left(\mathrm{H}_{2} / \mathrm{HI}\right)$ in galaxies are observed to follow radial profiles that are close to exponential functions (Leroy et al. 2008).

2. The localized $\mathrm{H}_{2} / \mathrm{H} \mathrm{I}$ in galaxies is predicted to depend on the midplane pressure, $P$, contributed mostly by the stars and the neutral gas. The correlation between $\mathrm{H}_{2} / \mathrm{H} \mathrm{I}$ and $P$ has been confirmed by observations (Leroy et al. 2008).

The original O09 model has the function form

$$
\begin{gathered}
\Sigma_{\mathrm{H} \mathrm{I}}(r)=\frac{\tilde{\Sigma}_{\mathrm{H}} \exp \left(-r / r_{\text {disk }}\right)}{1+R_{\mathrm{mol}}^{c} \exp \left(-1.6 r / r_{\mathrm{disk}}\right)}, \\
R_{\mathrm{mol}}^{c}=\left[K r_{\mathrm{disk}}^{-4} M_{\mathrm{gas}}\left(M_{\mathrm{gas}}+\left\langle f_{\sigma}\right\rangle M_{*}\right)\right]^{0.8},
\end{gathered}
$$

where $\tilde{\Sigma}_{\mathrm{H}}$ is the central gas surface density, $r_{\text {disk }}$ is the scale length of the gas profile, $R_{\mathrm{mol}}^{c}$ represents the central $\mathrm{H}_{2} / \mathrm{H} \mathrm{I}$ ratio, $M_{\text {gas }}=1.36\left(M_{\mathrm{H} \mathrm{I}, \text { tot }}+M_{\mathrm{H}_{2}}\right), \quad K \equiv 11.3 \mathrm{~m}^{4} \mathrm{~kg}^{-2}$, and $\left\langle f_{\sigma}\right\rangle \sim 0.4$.

Wang et al. (2014) used a similar model to Equation (2), but treated $\tilde{\Sigma}_{\mathrm{H}}$ and $R_{\mathrm{mol}}^{c}$ as free parameters to successfully fit the observed $\Sigma_{\mathrm{H} \text { I }}$ profiles of 39 galaxies. $\tilde{\Sigma}_{\mathrm{H}}$ is also adjustable in O09, but many assumptions have been made to produce Equation (3), including that $r_{\text {disk }}$ is twice the scale length of the stellar disk $\left(r_{\mathrm{s}}\right)$, and that the velocity dispersion is constant for the gas and exponentially rising as a function of radius for the stars.

Because $\mathrm{H} \mathrm{I}$ is on average more abundant and more extended than the $\mathrm{H}_{2}$ gas, we further assume $r_{\text {disk }}$ to be the scale length of the $\mathrm{H}$ I profile, so $r_{\text {disk }} \sim 0.2 R_{\mathrm{H} \text { I }}$ (W16, Wang et al. 2014). We hence can use the $\mathrm{O} 09$ model to guess the radial distribution of $\mathrm{H} \mathrm{I}, \Sigma_{\mathrm{H} \mathrm{I}}$ for ES2, based on the integrated $M_{\mathrm{H} \mathrm{I} \text {,tot }}, M_{\mathrm{H}_{2}}$, and $M_{*}$.

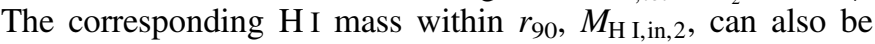
calculated.

\section{A.1.2. Modified O09 Models}

There are a few possible modifications to the original O09 model.

For the majority of galaxies from xGASS or ES2, which do not have $\mathrm{H}_{2}$ observations, we can approximate $M_{\mathrm{H}_{2}}=0.2 M_{\mathrm{H} \text { I,tot }}$, based on the average $\mathrm{H}_{2} / \mathrm{H} \mathrm{I}$ of galaxies with $M_{*}>10^{9} M_{\odot}$ (Saintonge et al. 2011). We note that $\mathrm{H}_{2} / \mathrm{H} \mathrm{I}$ increases as a function of $M_{*}$ (Catinella et al. 2018); however, as we will show later, using an accurate $\mathrm{H}_{2} / \mathrm{H}$ I does not significantly improve the method.

$r_{\text {disk }}$ need not necessarily be assumed to be $2 r_{\mathrm{s}}$, as it can be approximated as $0.2 R_{\mathrm{H}}$ (W16). Then, using a similar deduction procedure to $\mathrm{O} 09$, we obtain the following estimate of $R_{\mathrm{mol}}^{c}$ that can be used to replace Equation (3):

$$
R_{\mathrm{mol}}^{c}=\left(\Sigma_{\mathrm{gas}}\left(\Sigma_{\mathrm{gas}}+\Sigma_{*, \mathrm{eff}}\right)^{0.8} K^{0.8},\right.
$$

where

$$
\begin{gathered}
\Sigma_{\text {gas }}=M_{\text {gas }} / r_{\text {disk }}^{2} \exp \left(-r / r_{\text {disk }}\right), \\
\Sigma_{*, \text { eff }}=\left\langle f_{\sigma}\right\rangle / 4 M_{*} / r_{\mathrm{s}}^{2} \exp \left(-r / r_{\mathrm{s}}\right) .
\end{gathered}
$$

$\Sigma_{* \text {,eff }}$ can be viewed as the surface density of stars that effectively contribute to the midplane pressure, and hence to $R_{\mathrm{mol}}^{c}$.

\begin{tabular}{|c|c|c|c|c|}
\hline \multirow{3}{*}{ Method } & \multicolumn{2}{|c|}{$\underline{\log M_{\mathrm{H} \text { I,in,pred }} / M_{\mathrm{H} \mathrm{I,in}}}$} & \multirow{2}{*}{\multicolumn{2}{|c|}{$\frac{\Sigma_{\mathrm{H} \text { I,in,pred }}-\Sigma_{\mathrm{H} \text {, in }}}{\left(M_{\odot} \mathrm{pc}^{-2}\right)}$}} \\
\hline & \multirow[b]{2}{*}{ Median } & \multirow[b]{2}{*}{$\sigma$} & & \\
\hline & & & Median & $\sigma$ \\
\hline W16 & 0.06 & 0.09 & 0.82 & 1.37 \\
\hline O09-model 1 & 0.09 & 0.05 & 1.11 & 1.15 \\
\hline O09-model 2 & 0.10 & 0.05 & 1.14 & 1.10 \\
\hline O09-model 3 & 0.14 & 0.05 & 1.59 & 1.21 \\
\hline O09-model 4 & 0.14 & 0.06 & 1.70 & 1.20 \\
\hline
\end{tabular}

We predict $\Sigma_{\mathrm{H} \text { I }}$ and $M_{\mathrm{H} \text { I,in }}$ with the following modified 009 models for the galaxies from ES2:
Table 1

\begin{tabular}{|c|c|c|c|c|}
\hline \multirow{3}{*}{ Method } & \multicolumn{2}{|c|}{ 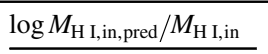 } & \multirow{2}{*}{\multicolumn{2}{|c|}{$\begin{array}{l}\Sigma_{\mathrm{H} \mathrm{I}, \text { in,pred }}-\Sigma_{\mathrm{H} \mathrm{I,in}} \\
\left(M_{\odot} \mathrm{pc}^{-2}\right)\end{array}$}} \\
\hline & \multirow[b]{2}{*}{ Median } & \multirow[b]{2}{*}{$\sigma$} & & \\
\hline & & & Median & $\sigma$ \\
\hline W16 & -0.01 & 0.09 & -0.04 & 0.60 \\
\hline O09-model 2 & 0.00 & 0.07 & -0.01 & 0.81 \\
\hline
\end{tabular}

Comparing Predicted $M_{\mathrm{HI} \text {,in }}$ and $\Sigma_{\mathrm{H} \text { I,in }}$ with Real Measurements in VS2

Table 2

Comparing Predicted $M_{\mathrm{H} \text { I,in }}$ and $\Sigma_{\mathrm{H} \mathrm{I,in}}$ with Real Measurements in VS

1. Model 2: $M_{\mathrm{H}_{2}}$ is replaced by $0.2 M_{\mathrm{H} \mathrm{I} \text {,tot }}$, and $R_{\mathrm{mol}}^{c}$ is estimated with Equation (3). This model requires only the input of $M_{\mathrm{H} \text {, tot }}$ and $M_{*}$, hence the minimum number of input parameters among the different models.

2. Model 3: $M_{\mathrm{H}_{2}}$ unchanged, and $R_{\mathrm{mol}}^{c}$ is estimated with Equation (4). This model uses real measurements of $M_{\text {gas }}$ and $r_{\mathrm{s}}$, and hence relies on fewer assumptions than the other three models.

3. Model 4: $M_{\mathrm{H}_{2}}$ is replaced by $0.2 M_{\mathrm{HI}}$,tot, and $R_{\mathrm{mol}}^{c}$ is estimated with Equation (4). This model requires inputting $M_{\mathrm{H} \text { I,tot }}, M_{*}$ and $r_{\mathrm{s}}$, which are in principle available for H I surveys like xGASS, which has optical images from SDSS.

\section{A.2. Selection among the Methods}

As in Section 3.1, we compare the estimated $M_{\mathrm{H} \text { I, in }}$ with the real measurements $M_{\mathrm{H} \text { I,in }} 0$ to assess the different methods. In addition to VS, we select the 10 THINGS galaxies from VS and call them VS2. These galaxies have CO (and hence the derived $\mathrm{H}_{2}$ ) images from BIMA SONG (Helfer et al. 2003) or HERACLES (Leroy et al. 2009). The scale lengths of the stellar disks $\left(r_{\mathrm{s}}\right)$ have been measured in Leroy et al. (2008). The availability of $\mathrm{H}_{2}$ images and $r_{\mathrm{s}}$ makes it possible to apply models 1,3 , and 4 of method O09.

We apply method W16 (which was presented in Section 3.1) and the different models of method O09 to ES2. We quantify the difference between the real and predicted amount of $\mathrm{HI}$ within $r_{90}, \log M_{\mathrm{H} \text { I,in,pred }} / M_{\mathrm{H} \text { I,in }}$ and $\Sigma_{\mathrm{H} \text { I,in,pred }}-\Sigma_{\mathrm{H} \text { I,in. The }}$ median and scatter (standard deviation) of the differences in ES2 with different methods are listed in Table 1. We note that the median absolute differences are less important than the scatters when assessing the performance of methods, for they can be calibrated (with ES2) and systematically removed later.

We first compare between the four models of method O09. Models 1 and 2 have smaller scatter, and hence work better than models 3 and 4. Model 2 uses the minimum number of input parameters, only $M_{\mathrm{H} \text { I,tot }}$ and $M_{*}$, hence the inclusion of $M_{\mathrm{H}_{2}}$ or $r_{\mathrm{s}}$ in the other three models does not seem to 

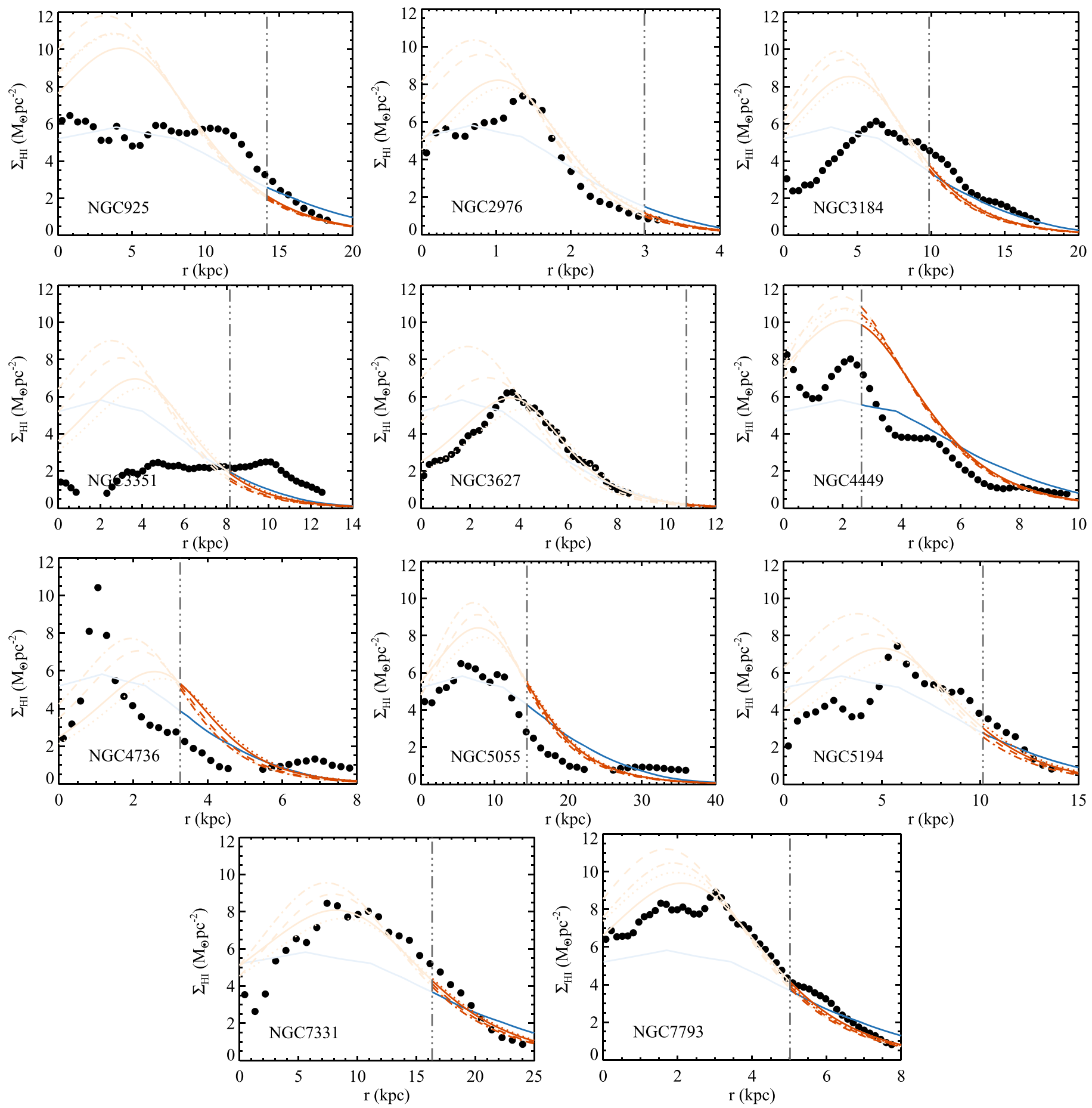

Figure 11. Real and predicted H I radial distributions. The black dots show the real measurements. The blue curves are predictions from method 1 . The orange dotted, solid, dashed, and dotted-dashed curves are predictions from models 1-4 of method 2 respectively. The black dashed-three-dotted lines mark the position of optical $r_{90}$.

significantly improve the predictions. It implies that due to the complexity of the $\mathrm{H}$ I-to- $\mathrm{H}_{2}$ process, the uncertainties of the models are large compared to the uncertainties of the input parameters. This is good news because $M_{\mathrm{H}_{2}}$ is only available for part of the xGASS sample and accurate measurements of $r_{\mathrm{s}}$ are tricky, due to the contamination of disk breaks, bars, and bulges (Gao \& Ho 2017). We hence choose model 2 among the four models of method 2 for the remaining analysis of this section, for it performs better than models 3 and 4 and requires fewer input parameters than model 1.
Within VS2, method W16 produces a slightly larger scatter in $\log M_{\mathrm{H} \mathrm{I,in,pred}} / M_{\mathrm{H} \text { I,in }}$ and $\Sigma_{\mathrm{H} \mathrm{I} \text {,in,pred }}-\Sigma_{\mathrm{H} \mathrm{I,in}}$ than model 2 of method O09. Considering the relatively small sample size of VS2, we further use VS to compare between method W16 and model 2. We have added -0.04 and -0.08 dex to the direct estimates of method W16 and model 2, respectively, to minimize the scatter and median offset from real measurements in VS.

The results of the comparison are displayed in Table 2 . Method W16 produces a slightly larger scatter in 


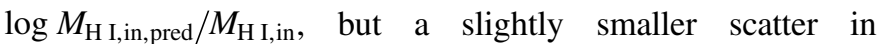
$\Sigma_{\mathrm{H} \text { I,in,pred }}-\Sigma_{\mathrm{H} \text { I,in }}$ than method O09. We further find with a figure close to Figure 3 that both methods produce $\log M_{\mathrm{H} \text { I,in,pred }} / M_{\mathrm{H} \text { I,in }}$ that do not significantly depend on $M_{*}$, $M_{\mathrm{H} \text { I,tot }} / M_{*}$, or sSFR. Putting these comparisons together, the two methods have similar performances in estimating $M_{\mathrm{H} \text {, in }}$ and $\Sigma_{\mathrm{H} \text { I,in }}$. We choose method W16 for its simplicity (only requiring $M_{\mathrm{HI}}$ tot as input, and model independent) for the analysis in this paper.

In case the readers might be interested, Figure 11 compares the predicted H I radial distributions to the real measurements in ES2. The models of method $\mathrm{O} 09$ match the real profiles in a remarkably close way in the outer regions, but much less closely in the inner regions. If we compare among the models of method O09, model 2 (orange solid curve) seems to provide the closest match to the $\Sigma_{\mathrm{H}}$ profiles within $r_{90}$, though physically it appears to be the most simplified model. The

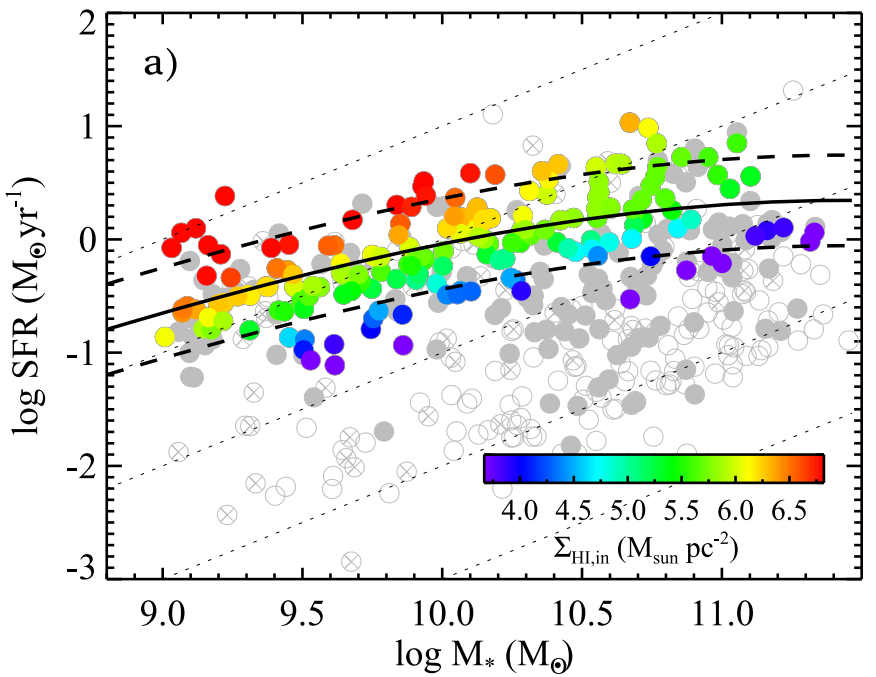

predicted profiles of method W16 never match the inner or outer profiles perfectly, but has been able to provide a reasonably close estimate to the $\mathrm{H}$ I mass and average surface densities in the inner regions.

\section{Appendix B}

Trends of $\boldsymbol{\Sigma}_{H}$ I,in when the Inner Disks are Defined by $\boldsymbol{R}_{25}$

There might be worry that using $r_{90}$ in the estimates of $M_{\mathrm{H} \text { I,in }}$ may cause systematic uncertainties due to the dependence of $r_{90}$ on the significance of bulges. We hence test this by replacing $r_{90}$ with $R_{25}$ in the estimate of $M_{\mathrm{H} \mathrm{I} \text {,in }}$, but find that all trends presented in the main part of this paper remain. We show two example plots in Figure 12, which are analogs of panel (e) of Figure 7 and panel (d) of Figure 9, but with the new $M_{\mathrm{H} \text { I,in }}$ estimates. We can see that the trends are similar with the two types of $M_{\mathrm{H} \text { I,in }}$ estimates.

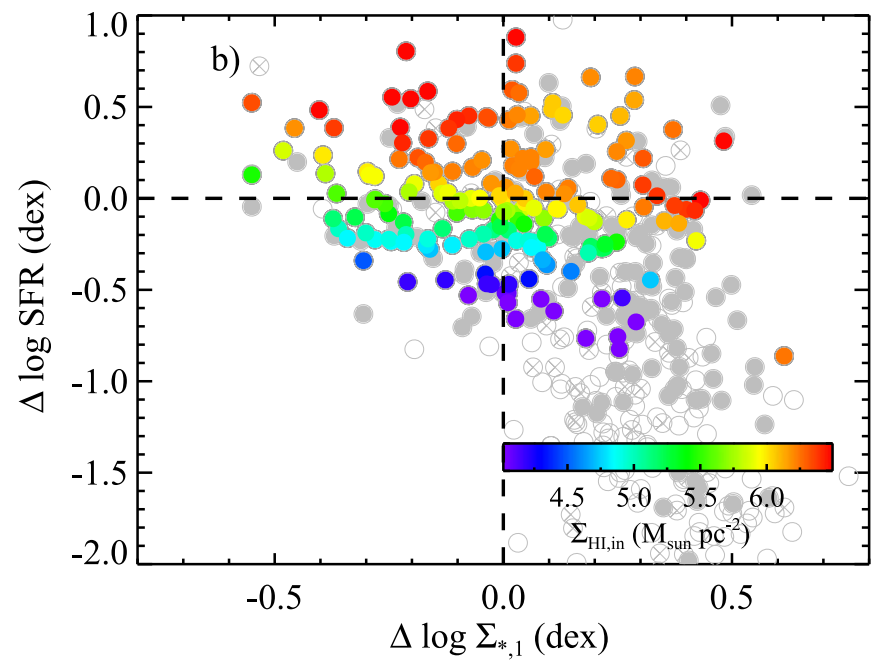

Figure 12. Left and right panels are similar to panel (e) of Figure 7 and panel (d) of Figure 9, respectively. The only difference is that $R_{25}$ instead of $r_{90}$ is used to estimate $M_{\mathrm{HI} \text {,in }}$. 


\section{ORCID iDs}

Barbara Catinella (i) https://orcid.org/0000-0002-7625-562X Amélie Saintonge (1) https://orcid.org/0000-0003-4357-3450 Zhizheng Pan (1) https://orcid.org/0000-0001-5662-8217

\section{References}

Abazajian, K. N., Adelman-McCarthy, J. K., Agüeros, M. A., et al. 2009, ApJS, 182, 543

Accurso, G., Saintonge, A., Catinella, B., et al. 2017, MNRAS, 470, 4750

Barro, G., Faber, S. M., Koo, D. C., et al. 2017, ApJ, 840, 47

Barro, G., Faber, S. M., Pérez-González, P. G., et al. 2013, ApJ, 765, 104

Barro, G., Faber, S. M., Pérez-González, P. G., et al. 2014, ApJ, 791, 52

Belfiore, F., Maiolino, R., Maraston, C., et al. 2017, MNRAS, 466, 2570

Berta, S., Lutz, D., Genzel, R., Förster-Schreiber, N. M., \& Tacconi, L. J. 2016, A\&A, 587, A73

Bigiel, F., Leroy, A., Walter, F., et al. 2008, AJ, 136, 2846

Bigiel, F., Leroy, A., Walter, F., et al. 2010, AJ, 140, 1194

Birnboim, Y., \& Dekel, A. 2003, MNRAS, 345, 349

Borthakur, S., Heckman, T., Tumlinson, J., et al. 2015, ApJ, 813, 46

Borthakur, S., Heckman, T., Tumlinson, J., et al. 2016, ApJ, 833, 259

Bournaud, F., Elmegreen, B. G., \& Elmegreen, D. M. 2007, ApJ, 670, 237

Brennan, R., Pandya, V., Somerville, R. S., et al. 2017, MNRAS, 465, 619

Brinchmann, J., Charlot, S., Kauffmann, G., et al. 2013, MNRAS, 432, 2112

Broeils, A. H., \& Rhee, M. H. 1997, A\&A, 324, 877

Brown, T., Catinella, B., Cortese, L., et al. 2017, MNRAS, 466, 1275

Bruce, V. A., Dunlop, J. S., McLure, R. J., et al. 2014, MNRAS, 444, 1001

Cacciato, M., Dekel, A., \& Genel, S. 2012, MNRAS, 421, 818

Cappellari, M., McDermid, R. M., Alatalo, K., et al. 2013, MNRAS, 432, 1862

Carton, D., Brinchmann, J., Wang, J., et al. 2015, MNRAS, 451, 210

Catinella, B., Saintonge, A., Janowiecki, S., et al. 2018, MNRAS, 476, 875

Catinella, B., Schiminovich, D., Cortese, L., et al. 2013, MNRAS, 436, 34

Catinella, B., Schiminovich, D., Kauffmann, G., et al. 2010, MNRAS, 403, 683

Chabrier, G. 2003, PASP, 115, 763

Charles, E. P. 2005, Psychological Methods, 10, 206

Cheung, E., Faber, S. M., Koo, D. C., et al. 2012, ApJ, 760, 131

Cortese, L., Catinella, B., \& Janowiecki, S. 2017, ApJL, 848, L7

de Blok, E., Fraternali, F., Heald, G., et al. 2015, in Advancing Astrophysics with the Square Kilometre Array (AASKA14) (Trieste: PoS), 129

Decarli, R., Walter, F., Gónzalez-López, J., et al. 2019, ApJ, 882, 138

Dekel, A., \& Burkert, A. 2014, MNRAS, 438, 1870

Dekel, A., Sari, R., \& Ceverino, D. 2009, ApJ, 703, 785

Elbaz, D., Dickinson, M., Hwang, H. S., et al. 2011, A\&A, 533, A119

Ellison, S. L., Sánchez, S. F., Ibarra-Medel, H., et al. 2018, MNRAS, 474, 2039

Fabello, S., Kauffmann, G., Catinella, B., et al. 2012, MNRAS, 427, 2841

Fang, J. J., Faber, S. M., Koo, D. C., \& Dekel, A. 2013, ApJ, 776, 63

Gabor, J. M., \& Davé, R. 2015, MNRAS, 447, 374

Gao, H., \& Ho, L. C. 2017, ApJ, 845, 114

Genzel, R., Tacconi, L. J., Lutz, D., et al. 2015, ApJ, 800, 20

Gil de Paz, A., Boissier, S., Madore, B. F., et al. 2007, ApJS, 173, 185

Giovanelli, R., Haynes, M. P., Kent, B. R., et al. 2005, AJ, 130, 2598

González Delgado, R. M., Cid Fernandes, R., Pérez, E., et al. 2016, A\&A, 590, A44

Grand, R. J. J., van de Voort, F., Zjupa, J., et al. 2019, MNRAS, 490, 4786

Groves, B. A., Schinnerer, E., Leroy, A., et al. 2015, ApJ, 799, 96

Haynes, M. P., Giovanelli, R., Kent, B. R., et al. 2018, ApJ, 861, 49

Haynes, M. P., Giovanelli, R., Martin, A. M., et al. 2011, AJ, 142, 170

Helfer, T. T., Thornley, M. D., Regan, M. W., et al. 2003, ApJS, 145, 259

Hess, K. M., \& Wilcots, E. M. 2013, AJ, 146, 124

Holwerda, B. W., Blyth, S. L., \& Baker, A. J. 2012, in IAU Symp. 284, The Spectral Energy Distribution of Galaxies-SED 2011, ed. R. J. Tuffs \& C. C. Popescu (Cambridge: Cambridge Univ. Press), 496

Huang, M.-L., \& Kauffmann, G. 2014, MNRAS, 443, 1329

Huang, M.-L., \& Kauffmann, G. 2015, MNRAS, 450, 1375

Huang, M.-L., Kauffmann, G., Chen, Y.-M., et al. 2013, MNRAS, 431, 2622 Janowiecki, S., Catinella, B., Cortese, L., et al. 2017, MNRAS, 466, 4795

Janowiecki, S., Cortese, L., Catinella, B., \& Goodwin, A. J. 2018, MNRAS, 476, 1390

Kauffmann, G., Heckman, T. M., White, S. D. M., et al. 2003, MNRAS, 341,33
Kennicutt, R. C. J. 1998, ARA\&A, 36, 189

Kennicutt, R. C. J., Armus, L., Bendo, G., et al. 2003, PASP, 115, 928

Kereš, D., Katz, N., Weinberg, D. H., \& Davé, R. 2005, MNRAS, 363, 2

Koribalski, B. S., Wang, J., Kamphuis, P., et al. 2018, MNRAS, 478, 1611

Krumholz, M. R. 2012, ApJ, 759, 9

Krumholz, M. R., Burkhart, B., Forbes, J. C., \& Crocker, R. M. 2018, MNRAS, 477, 2716

Leroy, A. K., Walter, F., Bigiel, F., et al. 2009, AJ, 137, 4670

Leroy, A. K., Walter, F., Brinks, E., et al. 2008, AJ, 136, 2782

Li, D., Wang, P., Qian, L., et al. 2018, IMMag, 19, 112

Luo, Y., Faber, S. M., Rodriguez-Puebla, A., et al. 2019, arXiv:1908.08055

Marasco, A., Fraternali, F., \& Binney, J. J. 2012, MNRAS, 419, 1107

Martinsson, T. P. K., Verheijen, M. A. W., Bershady, M. A., et al. 2016, A\&A, 585, A99

Meyer, M. 2009, in Panoramic Radio Astronomy: Wide-field 1-2GHz Research on Galaxy Evolution, ed. G. Heald \& P. Serra (Groningen: PoS), 15

Mo, H. J., Mao, S., \& White, S. D. M. 1998, MNRAS, 295, 319

Moran, S. M., Heckman, T. M., Kauffmann, G., et al. 2012, ApJ, 745, 66

Moran, S. M., Kauffmann, G., Heckman, T. M., et al. 2010, ApJ, 720, 1126

Morrissey, P., Conrow, T., Barlow, T. A., et al. 2007, ApJS, 173, 682

Mosleh, M., Tacchella, S., Renzini, A., et al. 2017, ApJ, 837, 2

Nelson, E., van Dokkum, P., Franx, M., et al. 2014, Natur, 513, 394

Noeske, K. G., Weiner, B. J., Faber, S. M., et al. 2007, ApJL, 660, L43

Noguchi, M. 1998, Natur, 392, 253

Noordermeer, E., van der Hulst, J. M., Sancisi, R., Swaters, R. A., \& van Albada, T. S. 2005, A\&A, 442, 137

Obreschkow, D., Croton, D., De Lucia, G., Khochfar, S., \& Rawlings, S. 2009, ApJ, 698, 1467

Putman, M. E. 2017, ASSL, 430, 1

Rees, M. J., \& Ostriker, J. P. 1977, MNRAS, 179, 541

Saintonge, A., Catinella, B., Cortese, L., et al. 2016, MNRAS, 462, 1749

Saintonge, A., Catinella, B., Tacconi, L. J., et al. 2017, ApJS, 233, 22

Saintonge, A., Kauffmann, G., Kramer, C., et al. 2011, MNRAS, 415, 32

Saintonge, A., Tacconi, L. J., Fabello, S., et al. 2012, ApJ, 758, 73

Schiminovich, D., Catinella, B., Kauffmann, G., et al. 2010, MNRAS, 408, 919

Schiminovich, D., Wyder, T. K., Martin, D. C., et al. 2007, ApJS, 173, 315

Schruba, A., Leroy, A. K., Walter, F., et al. 2011, AJ, 142, 37

Scoville, N., Sheth, K., Aussel, H., et al. 2016, ApJ, 820, 83

Silverman, J. D., Daddi, E., Rodighiero, G., et al. 2015, ApJL, 812, L23

Speagle, J. S., Steinhardt, C. L., Capak, P. L., \& Silverman, J. D. 2014, ApJS, 214,15

Springob, C. M., Haynes, M. P., Giovanelli, R., \& Kent, B. R. 2005, ApJS, 160,149

Staveley-Smith, L., \& Oosterloo, T. 2015, in Advancing Astrophysics with the Square Kilometre Array (AASKA14) (Trieste: PoS), 167

Swaters, R. A., \& Balcells, M. 2002, A\&A, 390, 863

Swaters, R. A., van Albada, T. S., van der Hulst, J. M., \& Sancisi, R. 2002, A\&A, 390, 829

Tacchella, S., Carollo, C. M., Renzini, A., et al. 2015, Sci, 348, 314

Tacchella, S., Dekel, A., Carollo, C. M., et al. 2016, MNRAS, 458, 242

Tacconi, L. J., Genzel, R., Saintonge, A., et al. 2018, ApJ, 853, 179

Tacconi, L. J., Neri, R., Genzel, R., et al. 2013, ApJ, 768, 74

van de Voort, F., Schaye, J., Booth, C. M., Haas, M. R., \& Dalla Vecchia, C. 2011, MNRAS, 414, 2458

Verheijen, M. A. W., \& Sancisi, R. 2001, A\&A, 370, 765

Walter, F., Brinks, E., de Blok, W. J. G., et al. 2008, AJ, 136, 2563

Wang, E., Kong, X., \& Pan, Z. 2018, ApJ, 865, 49

Wang, J., Fu, J., Aumer, M., et al. 2014, MNRAS, 441, 2159

Wang, J., Kauffmann, G., Józsa, G. I. G., et al. 2013, MNRAS, 433, 270

Wang, J., Kauffmann, G., Overzier, R., et al. 2011, MNRAS, 412, 1081

Wang, J., Koribalski, B. S., Jarrett, T. H., et al. 2017, MNRAS, 472, 3029

Wang, J., Koribalski, B. S., Serra, P., et al. 2016, MNRAS, 460, 2143

Wenger, M., Ochsenbein, F., Egret, D., et al. 2000, A\&AS, 143, 9

Whitaker, K. E., Bezanson, R., van Dokkum, P. G., et al. 2017, ApJ, 838, 19

Whitaker, K. E., Franx, M., Leja, J., et al. 2014, ApJ, 795, 104

Whitaker, K. E., van Dokkum, P. G., Brammer, G., \& Franx, M. 2012, ApJL, 754, L29

White, S. D. M., \& Frenk, C. S. 1991, ApJ, 379, 52

Williams, C. C., Giavalisco, M., Cassata, P., et al. 2014, ApJ, 780, 1

Woo, J., Dekel, A., Faber, S. M., \& Koo, D. C. 2015, MNRAS, 448, 237

Wright, E. L., Eisenhardt, P. R. M., Mainzer, A. K., et al. 2010, AJ, 140, 1868 
Yang, X., Mo, H. J., van den Bosch, F. C., et al. 2007, ApJ, 671, 153

Yesuf, H. M., \& Ho, L. C. 2019, ApJ, 884, 177

Yıldız, M. K., Serra, P., Peletier, R. F., Oosterloo, T. A., \& Duc, P.-A. 2017, MNRAS, 464, 329
Yim, K., \& van der Hulst, J. M. 2016, MNRAS, 463, 2092

Zhang, K., Wu, J., Li, D., et al. 2019, SCPMA, 62, 959506

Zibetti, S., Charlot, S., \& Rix, H.-W. 2009, MNRAS, 400, 1181

Zolotov, A., Dekel, A., Mandelker, N., et al. 2015, MNRAS, 450, 2327 